\title{
Verifying a Quantitative Relaxation of Linearizability via Refinement
}

\author{
Kiran Adhikari \\ Thesis submitted to the Faculty of the \\ Virginia Polytechnic Institute and State University \\ in partial fulfillment of the requirements for the degree of \\ Master of Science \\ in \\ Computer Engineering \\ Chao Wang, Chair \\ Patrick Schaumont \\ Michael Hsiao
}

May 6, 2013

Blacksburg, Virginia

Keywords: Quasi Linearizability, Refinement, Model Checking Copyright 2013, Kiran Adhikari 


\title{
Verifying a Quantitative Relaxation of Linearizability via Refinement
}

\author{
Kiran Adhikari
}

(ABSTRACT)

Concurrent data structures have found increasingly widespread use in both multicore and distributed computing environments, thereby escalating the priority for verifying their correctness. The thread safe behavior of these concurrent objects is often described using formal semantics known as linearizability, which requires that every operation in a concurrent object appears to take effect between its invocation and response. Quasi linearizability is a quantitative relaxation of linearizability to allow more implementation freedom for performance optimization. However, ensuring the quantitative aspects of this new correctness condition is an arduous task. We propose the first method for formally verifying quasi linearizability of the implementation model of a concurrent data structure. The method is based on checking the refinement relation between the implementation model and a specification model via explicit state model checking. It can directly handle multi-threaded programs where each thread can make infinitely many method calls, without requiring the user to manually annotate for the linearization points. We have implemented and evaluated our method in the PAT model checking toolkit. Our experiments show that the method is effective in verifying quasi linearizability and in detecting its violations. 


\section{Dedication}

I dedicate this thesis to my grandfather, my parents and my brother. 


\section{Acknowledgment}

I extend my gratitude to my advisor, Professor Chao Wang for his consistent guidance and continuous support throughout my research. His advice and encouragement have been extremely valuable in helping me complete my thesis. I would also like to thank Dr. Michael Hsiao and Dr. Patrick Schaumont for serving as members of my thesis committee.

I sincerely thank my seniors Sanjay Basnet, Gyanendra Shrestha, Nabin Jnawali, Ram Thapa and Bipin Jnawali for their consistent encouragement. For all my friends, including Diwas, Suju, Krishna, Keshab, Prakash and Suwarna, in particular thank you for your direct and indirect support and your company throughout the years. I am also deeply grateful to James Street, whose help was very beneficial to this thesis and I really enjoyed working together with him.

I am deeply indebted to my beloved grandfather, my parents and all the other family members, for their endless love and support. They have always been there for me, through thick and thin.

Kiran Adhikari

May 6, 2013 


\section{Contents}

List of Figures

viii

List of Tables $\quad x$

1 Introduction 1

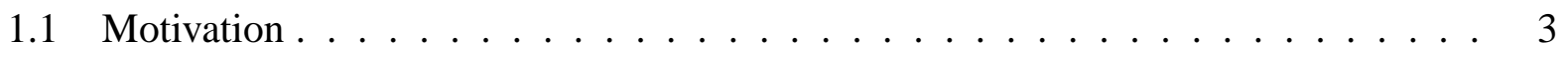

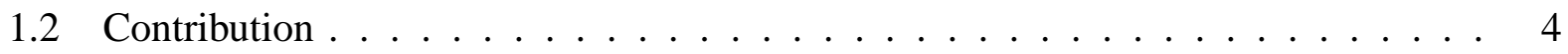

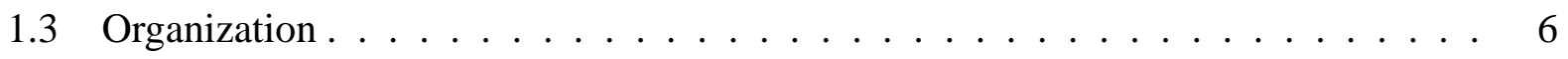

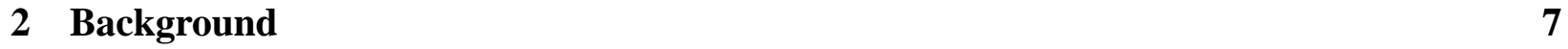

2.1 Linearizability . . . . . . . . . . . . . . . . 8

2.2 Quasi Linearizability . . . . . . . . . . . . . . . . . . . 10

2.3 Linearizability as Refinement . . . . . . . . . . . . . . . . . . . 14 
2.4 Realization of a Model . . . . . . . . . . . . . . . . . 17

2.5 Related Work . . . . . . . . . . . . . . . . . 18

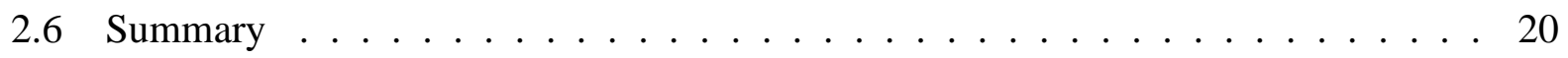

3 Verifying Quasi Linearizability via Refinement Checking 21

3.1 Two Approaches of Verification . . . . . . . . . . . . . . . 22

3.2 The Manual Verification Approach . . . . . . . . . . . . . . . . . 24

3.2.1 Specification Model $M_{\text {spec }} \ldots \ldots \ldots \ldots \ldots$

3.2.2 Implementation Model $M_{i m p l}: \ldots \ldots \ldots \ldots$

3.2.3 Relaxed Specification Model $M_{\text {spec }}^{\prime} \ldots \ldots \ldots$. . . . . . . . . . 28

3.2.4 Checking Refinement Relation: . . . . . . . . . . . . . . . . 29

3.3 Experimental Results . . . . . . . . . . . . . . . . . 30

3.4 Limitations of the Manual Approach . . . . . . . . . . . . . . . . 32

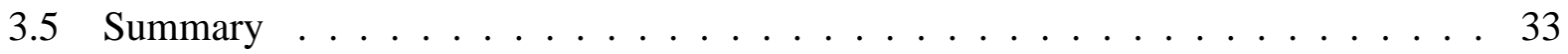

4 New Algorithm for Checking the Quasi Refinement Relation 34

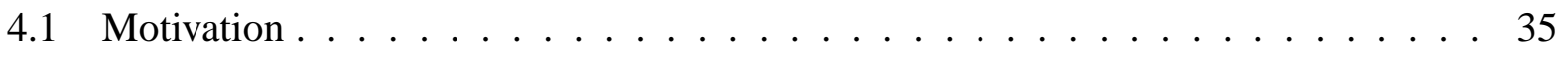

4.2 Linearizability Checking via Quasi Refinement . . . . . . . . . . . . . 35 
4.3 Generation of Relaxed Specification . . . . . . . . . . . . . 38

4.4 Experimental Results . . . . . . . . . . . . . . . . . . . 43

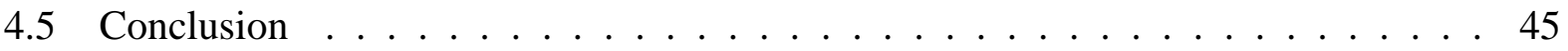

5 Conclusions and the Future Work 46

$\begin{array}{ll}\text { Bibliography } & 48\end{array}$ 


\section{List of Figures}

2.1 Execution traces of a queue. $\ldots \ldots \ldots \ldots \ldots$

2.2 An LTS example . . . . . . . . . . . . . . . . . . . . . . . 15

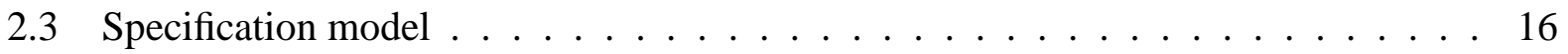

2.4 Implementation model $\ldots \ldots \ldots \ldots \ldots \ldots$

3.1 Verifying quasi linearizability: manual approach (left) and automated approach

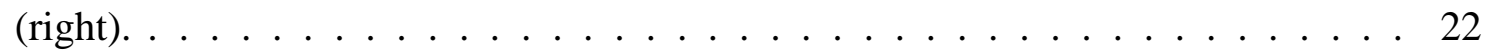

3.2 Implementations of a 4 -quasi queue $\ldots \ldots \ldots \ldots \ldots \ldots$

3.3 Valid histories of a 1-quasi linearizable queue . . . . . . . . . . . . . 26

3.4 Graph showing the linearizability checking time for different QFs and segment

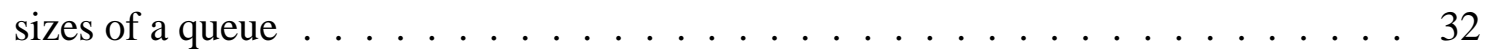

4.1 Specification model before adding relaxed transitions for state $s_{1} \ldots \ldots \ldots$. . . 39 
4.2 Specification model after adding relaxed edges for state $s_{1}$ and quasi factor $1 . \quad$. . 39 


\section{List of Tables}

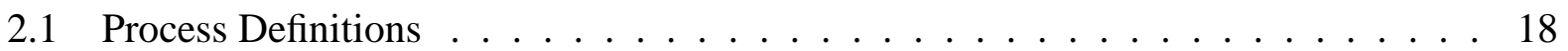

3.1 Experimental results for standard refinement checking. . . . . . . . . . . . 31

4.1 Specification sequence generation at state $s_{1} \ldots \ldots \ldots$

4.2 Statistics of the benchmark examples . . . . . . . . . . . . . . . 43

4.3 Results for checking quasi linearizability with 2 threads . . . . . . . . . . . . . 44 


\section{List of Abbreviations}

$\begin{array}{ll}\text { BFS } & \text { Breadth-First Search } \\ \text { DFS } & \text { Depth-First Search } \\ \text { PAT } & \text { Process Analysis Toolkit } \\ \text { CSP } & \text { Communicating Sequential Processes } \\ \text { LTS } & \text { Labeled Transition System } \\ \text { QF } & \text { Quasi Factor }\end{array}$




\section{Chapter 1}

\section{Introduction}

Concurrent programming has engulfed the broad horizon of both the multi-core and distributed computing environments. As Moore's law starts to pay little dividend in improving the performance of sequential applications, programmers need to adopt the concurrent programming paradigm to help reduce the memory contention and improve the scalability and throughput of the applications significantly. This has led to the increasingly widespread use of concurrent data structures in domains spanning from embedded computing to distributed systems. However, it is often difficult to build and test the thread safe components for the concurrent programming environment without placing undue synchronization overhead. The often large number of thread interleavings, along with the subtle interactions of concurrent operations, makes it difficult to obtain the correct implementations. This has escalated the priority for verifying the correctness of these concurrent data structures. 
Over the past two decades, researchers have focused on using linearizability as a correctness condition for concurrent data structures. A concurrent data structure is linearizable if each of its operations or method calls appears to take effect instantaneously at some point in time between its invocation and response. Although being linearizable does not necessarily ensure the fullfledged correctness of the implementation, linearizability violations are clear indicators that the implementation is buggy. In this sense, linearizability serves as a useful criterion for implementing concurrent data structures. However, ensuring linearizability of highly concurrent data structures is a difficult task, due to the subtle interactions of concurrent operations and the often astronomically many interleavings.

Although linearizability is non-blocking[10], it often imposes unnecessarily tight synchronization requirement on the implementation and therefore limit the performance and scalability. Realizing that a more relaxed correctness condition than linearizability suffices in many applications, Afek et al. [1] have defined a new notion called quasi linearizability. Quasi linearizability is a quantitative relaxation of linearizability $[9,12,17]$ to allow for more flexibility in how the data structures are implemented. While preserving the basic intuition of linearizability, quasi linearizability relaxes the semantics of the data structures to achieve increased runtime performance. For example, when implementing a queue for a task scheduler in a thread pool, it is often the case that we do not need the strict first-in-first-out (FIFO) semantics; instead, we may want to allow the dequeue operations to be overtaken occasionally if it helps improving the runtime performance. The only requirement here is that such out-of-order execution should be bounded by a fixed number of steps. Similarly, when implementing data caching in web applications, we may not need the strict semantics of 
standard data structures, since getting stale data occasionally is acceptable as long as the delay is bounded. Furthermore in distributed systems, the counter for generating unique identifiers may also be allowed to return out-of-order values occasionally.

\subsection{Motivation}

Despite the advantages of quasi linearizability and its rising popularity (e.g., $[9,12,17])$, such relaxed consistency property is difficult to test and verify. Inspit of being an important property of concurrent data structures, there does not yet exist any effective way of formally verifying this relaxed correctness condition.

Although there is a large body of work on formally verifying standard linearizability, for example, the methods based on model checking $[15,14,24,5]$, runtime verification [4], and mechanical proofs $[22,23]$, they cannot directly verify quasi linearizability. Because of the inherent nondeterminism in the quasi linearizable data structures, these traditional verification methods do not have the capability of checking teh quasi linearizability semantics. In addition to the requirement of covering all possible interleavings of concurrent events, one needs to accurately analyze the quantitative aspects of these interleavings to verify quasi linearizability. Another reason why formal verification methods for quasi linearizability are lacking is because quasi linearizability is relatively new and still under development.

In this thesis, we fill the gap by developing an effecient method to verify this quantitative relaxation of the linearizability for concurrent data structures. 


\subsection{Contribution}

We propose the first automated method for formally verifying quasi linearizability in the implementation models of concurrent data structures. There are several technical challenges. First, since the number of concurrent operations in each thread is unbounded, the execution trace of a multithreaded program that uses the concurrent data structures may be infinitely long. This precludes the use of existing methods such as LineUp [4] because they are based on checking permutations of finite length execution histories. Second, since the method needs to be fully automated, we do not assume that the user will provide hints or annotate the linearization points of each method. This precludes the use of existing methods that are based on either user guidance (e.g., [22, 23]) or annotated linearization points (e.g., [24]).

To overcome these challenges, we rely on explicit state model checking. That is, given an implementation model $M_{i m p l}$ and a specification model $M_{s p e c}$, we check whether the set of execution traces of $M_{i m p l}$ is a subset of the execution traces of $M_{\text {spec }}$. Toward this end, we extend a classic refinement checking algorithm so that it can check for the newly defined quantitative relaxation of standard refinement relation.

Consider a quasi linearizable queue as an example. Starting from the pair of initial states of a FIFO queue specification model and its quasi linearizable implementation model, we check whether all subsequent state transitions of the implementation model can match some subsequent state transitions of the specification model. To make sure that the verification problem remains decidable, we bound the capacity of the data structure in the model, to ensure that the number of 
states of the program is finite.

We have implemented the new method in the Process Analysis Toolkit (PAT) [20], which is a model checker for analysing concurrent systems. PAT provides the basic infrastructure for parsing and analyzing the specification and implementation models written in a process algebra language that resembles Tony Hoare's Communicating Sequential Processes (CSP) [11]. Our new method is implemented as a module in PAT, and is compared against the existing module for checking standard refinement relation. Our experiments on a set of popular concurrent datastructures, such as queues, stacks or priority queues show that the new method is effective in detecting subtle violations of quasi linearizability. When the implementation model is indeed correct, our method can also generate the proof of correctness quickly.

To sum up, this thesis make the following contributions:

- Propose the first method for formally verifying quasi linearizability of concurrent data structures. This is accomplished by designing a new algorithm for checking a relaxed version of the refinement relation between the implementation and specification models.

- Implement the new method in a software tool called PAT and demonstrate its effectiveness on a set of quasi linearizable concurrent data structure examples including queues, stacks, and priority queues. 


\subsection{Organization}

The remainder of this thesis is organized as follows.

Chapter 2 provides the overview of the notations and review the existing refinement checking algorithm for verifying the standard linearizability.

Chapter 3 introduces the overall flow of our new relaxed linearizability checking method. This chapter also presents a manual approach for verifying quasi linearizability based on the standard refinement checking algorithm. This approach is proved to be labor intensive and error prone, therefore motivating us to design a fully automated method.

Chapter 4 presents the fully automated method, which is based on a new algorithm for checking the relaxed refinement relation. This chapter also presents the experimental results.

Chapter 5 gives the conclusions and outlines the future works. 


\section{Chapter 2}

\section{Background}

In this chapter, the notion of linearizability and quasi linearizability are defined by using the refinement relations between an implementation model and the specification model. We also present the process algera semantics of the models in relation to the CSP. Finally, we introduce the standard refinement checking algorithm as an approach to verify the linearizability. The method relies on model checking of finite state systems specified as concurrent processes with shared variables and take advantage of a new trace refinement checking to verify the relaxed version of linearizable data structures.

Given a two labeled transition systems, the following section explains the formal semantics defining standard and quasi linearizability, and review an existing algorithm for checking the refinement relation between those two labeled transition systems. 


\subsection{Linearizability}

Linearizability [10] is a safety property of concurrent systems, over sequences of actions corresponding to the invocations and responses of the operations on shared objects. We begin by formally defining the shared memory model.

Definition 1 (System Models). A shared memory model $\mathcal{M}$ is a 3-tuple structure $\left(O\right.$, init $\left._{O}, P\right)$, where $O$ is a finite set of shared objects, init ${ }_{O}$ is the initial valuation of $O$, and $P$ is a finite set of processes accessing the objects.

Every shared object has a set of states. Each object supports a set of operations, which are pairs of invocations and matching responses. These operations are the only means of accessing the state of the object. A shared object is deterministic if, given the current state and an invocation of an operation, the next state of the object and the return value of the operation are unique. Otherwise, the shared object is non-deterministic. A sequential specification ${ }^{1}$ of a deterministic (resp. nondeterministic) shared object is a function that maps every pair of invocation and object state to a pair (resp. a set of pairs) of response and a new object state.

An execution of the shared memory model $\mathcal{M}=\left(O\right.$, init $\left._{O}, P\right)$ is modeled by a history, which is a sequence of operation invocations and response actions that can be performed on $O$ by processes in $P$. The behavior of $\mathcal{M}$ is defined as the set, $H$, of all possible histories together. A history $\sigma$ $\in H$ induces an irreflexive partial order $<_{\sigma}$ on operations such that $o p_{1}<_{\sigma} o p_{2}$ if the response of operation $o p_{1}$ occurs in $\sigma$ before the invocation of operation $o p_{2}$. Operations in $\sigma$ that are not

\footnotetext{
${ }^{1}$ More rigorously, the sequential specification is for a type of shared objects. For simplicity, however, we refer to both actual shared objects and their types interchangeably in this paper.
} 
related by $<_{\sigma}$ are concurrent. A history $\sigma$ is sequential iff $<_{\sigma}$ is a strict total order.

Let $\left.\sigma\right|_{i}$ be the projection of $\sigma$ on process $p_{i}$, which is the subsequence of $\sigma$ consisting of all invocations and responses that are performed by $p_{i}$ in $P$. Let $\left.\sigma\right|_{o_{i}}$ be the projection of $\sigma$ on object $o_{i}$ in $O$, which is the subsequence of $\sigma$ consisting of all invocations and responses of operations that are performed on object $o_{i}$. Every history $\sigma$ of a shared memory model $\mathcal{M}=\left(O\right.$, init $\left._{O}, P\right)$ must satisfy the following basic properties:

- Correct interaction: For each process $p_{i} \in P,\left.\sigma\right|_{i}$ consists of alternating invocations and matching responses, starting with an invocation. This property prevents pipelining ${ }^{2}$ operations.

- Closedness ${ }^{3}$ : Every invocation has a matching response. This property prevents pending operations.

A sequential history $\sigma$ is legal if it respects the sequential specifications of the objects. More specifically, for each object $o_{i}$, there exists a sequence of states $s_{0}, s_{1}, s_{2}, \ldots$ of object $o_{i}$, such that $s_{0}$ is the initial valuation of $o_{i}$, and for all $j=1,2, \ldots$ according to the sequential specification (the function), the $j$-th invocation in $\left.\sigma\right|_{o_{i}}$ together with state $s_{j-1}$ will generate the $j$-th response in $\left.\sigma\right|_{o_{i}}$ and state $s_{j}$. For example, a sequence of read and write operations of an object is legal if each read returns the value of the preceding write if there is one, and otherwise it returns the initial

\footnotetext{
${ }^{2}$ Pipelining operations mean that after invoking an operation, a process invokes another (same or different) operation before the response of the first operation.

${ }^{3}$ This property is not required in the original definition of linearizability in [10]. However adding it will not affect the correctness of our result because by Theorem 2 in [10], for a pending invocation in a linearizable history, we can always extend the history to a complete one and preserve linearizability. We include this property to obviate the discussion for pending invocations.
} 
value.

Given a history $\sigma$, a sequential permutation $\pi$ of $\sigma$ is a sequential history in which the set of operations as well as the initial states of the objects are the same as in $\sigma$.

Definition 2 (Linearizability). Given a model $\mathcal{M}=\left(O=\left\{o_{1}, \ldots, o_{k}\right\}\right.$, init $\left.{ }_{O}, P=\left\{p_{1}, \ldots, p_{n}\right\}\right)$. Let $H$ be the behavior of $\mathcal{M} . \mathcal{M}$ is linearizable iffor any history $\sigma$ in $H$, there exists a sequential permutation $\pi$ of $\sigma$ such that

1. for each object $o_{i}(1 \leq i \leq k),\left.\pi\right|_{o_{i}}$ is a legal sequential history (i.e., $\pi$ respects the sequential specification of the objects), and

2. for every $o p_{1}$ and $o p_{2}$ in $\sigma$, if $o p_{1}<_{\sigma} o p_{2}$, then $o p_{1}<_{\pi}$ op $p_{2}$ (i.e., $\pi$ respects the run-time ordering of operations).

Linearizability can be equivalently defined as follows. In every history $\sigma$, if we assign increasing time values to all invocations and responses, then every operation can be shrunk to a single time point between its invocation time and response time such that the operation appears to be completed instantaneously at this time point $[16,3]$. This time point is called its linearization point.

\subsection{Quasi Linearizability}

For two histories $\sigma$ and $\sigma^{\prime}$ such that one is the permutation of the other, we define their distance as follows. Let $\sigma=e_{1}, e_{2}, e_{3}, \ldots, e_{n}$ and $\sigma^{\prime}=e_{1}^{\prime}, e_{2}^{\prime}, e_{3}^{\prime}, \ldots, e_{n}^{\prime}$. Let $\sigma[e]$ and $\sigma^{\prime}[e]$ be the indices 
of the event $e$ in histories $\sigma$ and $\sigma^{\prime}$, respectively. The distance between the two histories, denoted $\Delta\left(\sigma, \sigma^{\prime}\right)$, is defined as follows:

$$
\Delta\left(\sigma, \sigma^{\prime}\right)=\max _{e \in \sigma}\left\{\left|\sigma^{\prime}[e]-\sigma[e]\right|\right\} .
$$

In other words, the distance between $\sigma$ and $\sigma^{\prime}$ is the maximum distance that an event in $\sigma$ has to move to arrive at its position in $\sigma^{\prime}$.

While measuring the distance between two histories, we often care about only a subset of method calls. For example, in a concurrent queue, we may care about the ordering of enqueue and dequeue operations while ignoring calls to size operation. In the remaining of this work, we use words enq and deq for the interests of space. Furthermore, we may allow deq operations to be executed out of order, but keep enq operations in order. In such case, we need a way to add ordering constraints on a subset of the methods of the shared object.

Let $\operatorname{Domain}(o)$ be the set of all operations of a shared object $o$. Let $d \subset \operatorname{Domain}(o)$ be a subset of operations. Let Powerset(Domain(o)) be the set of all subsets of Domain(o). Let $D \subset$ Powerset $(\operatorname{Domain}(o))$ be a subset of the powerset.

Definition 3 (Quasi Linearization Factor). A quasi-linearization factor is a function $Q_{O}: D \rightarrow \mathbb{N}$, where $D$ is a subset of the powerset and $\mathbb{N}$ is the set of natural numbers.

Example 1. For a bounded queue that stores a set $X$ of non-zero data items, we have Domain (queue) = $\{$ enq.x, deq.x, deq. $0 \mid x \in X\}$, where enq.x denotes the enqueue operation for data $x$, deq.x denotes the dequeue operation for data $x$, and deq.0 indicates that the queue is empty. We may 
define two subsets of Domain(queue):

$$
\begin{aligned}
& d_{1}=\{\text { enq.y } \mid y \in Y\}, \\
& d_{2}=\{\text { deq.y } \mid y \in Y\} .
\end{aligned}
$$

Let $D=\left\{d_{1}, d_{2}\right\}$, where $d_{1}$ is the subset of deq events and $d_{2}$ is the subset of enq events. The distance between $\sigma$ and $\sigma^{\prime}$, after being projected to subsets $d_{1}$ and $d_{2}$, is defined as $\Delta\left(\left.\sigma\right|_{d_{1}},\left.\sigma^{\prime}\right|_{d_{2}}\right)$. If we require that the enq calls follow the FIFO order and the deq calls be out-of-order by at most $K$ steps, the quasi-linearization factor $Q_{\{\text {queue }}: D \rightarrow \mathbb{N}$ is defined as follows:

$$
\begin{aligned}
& Q_{\{\text {queue }\}}\left(d_{1}\right)=0, \\
& Q_{\{\text {queue }}\left(d_{2}\right)=K .
\end{aligned}
$$

Definition 4 (Quasi Linearizability). Given a model $\mathcal{M}=\left(O=\left\{o_{1}, \ldots, o_{k}\right\}\right.$, init ${ }_{O}, P=$ $\left.\left\{p_{1}, \ldots, p_{n}\right\}\right)$. Let $H$ be the behavior of $\mathcal{M}$. $\mathcal{M}$ is quasi linearizable under the quasi factor $Q_{O}: D \rightarrow \mathbb{N}$ if for any history $\sigma$ in $H$, there exists a sequential permutation $\pi$ of $\sigma$ such that

- for every $o p_{1}$ and $o p_{2}$ in $\sigma$, if $o p_{1}<_{\sigma}$ op $p_{2}$, then $o p_{1}<_{\pi}$ op $p_{2}$ (i.e., $\pi$ respects the run-time ordering of operations), and

- for each object $o_{i}(1 \leq i \leq k)$, there exists another sequential permutation $\pi^{\prime}$ of $\pi$ such that

1. $\left.\pi^{\prime}\right|_{o_{i}}$ is a legal sequential history (i.e., $\pi^{\prime}$ respects the sequential specification of the objects) and

2. $\Delta\left(\left.\left(\left.\pi\right|_{o_{i}}\right)\right|_{d},\left.\left(\left.\pi^{\prime}\right|_{o_{i}}\right)\right|_{d}\right) \leq Q_{O}(d)$ for all $d \in D$. 
This definition subsumes the definition for linearizability because, if the quasi factor is $Q_{O}(d)=0$ for all $d \in D$, then the objects behaves as a standard linearizable data structure, e.g., a FIFO queue.

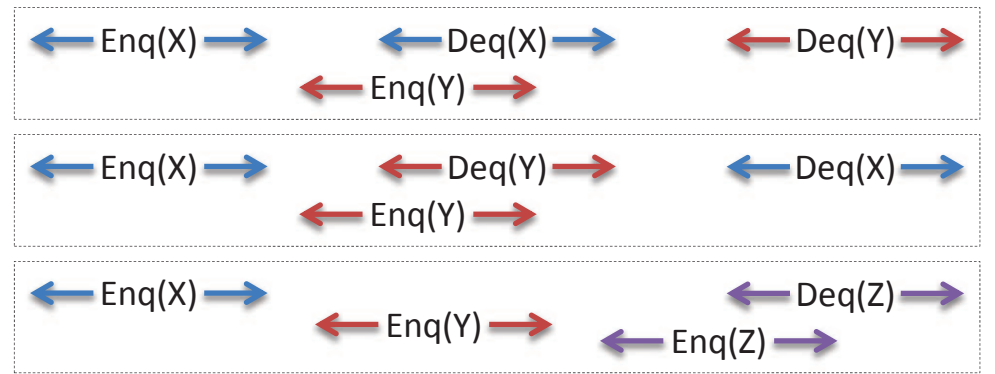

Only the first trace (at the top) is linearizable. The second trace is not linearizable, but is 1-quasi linearizable. The third trace is only 2-quasi linearizable.

Figure 2.1: Execution traces of a queue.

Example 2. Consider the concurrent execution of a queue as shown in the Fig. 2.1. In the first part, it is clear that the execution is linearizable, because it is a valid permutation of the sequential history where Enq(Y) takes effect before Deq (X). The second part is not linearizable, because the first dequeue operation is Deq(Y) but the first enqueue operation is Enq(X). However, it is interesting to note that the second history is not far from a linearizable history, since swapping the order of the two dequeue events would make it linearizable. Therefore, flexibility is provided in dequeue events to allow them to be reordered. Similarly, for the third part, if the quasi factor is 0 (no out-of-order execution) or 1 (out-of-order by at most 1 step), then the history is not quasi linearizable. However, if the quasi factor is 2 (out-of-order by at most 2 steps), then the third history in Fig.2.1 is considered as quasi linearizable. 


\subsection{Linearizability as Refinement}

Linearizability is defined in terms of the invocations and responses of high-level operations. In a real concurrent program, the high-level operations are implemented by algorithms on concrete shared data structures, e.g., a linked list that implements a shared stack object [21]. Therefore, the execution of high-level operations may have complicated interleaving of low-level actions. Linearizability of a concrete concurrent algorithm requires that, despite low-level interleaving, the history of high-level invocation and response actions still has a sequential permutation that respects both the run-time ordering among operations and the sequential specification of the objects.

For verifying standard (but not quasi) linearizability, an existing method $[15,14]$ can be used to check whether a real concurrent algorithm (we refer as implementation in this work) refines the high-level linearizable requirement (we refer as specification in this work). In this case, the behaviors of the implementation and the specification are modeled as labeled transition systems (LTSs), and the refinement checking is accomplished by using explicit state model checking.

Definition 5 (Labeled Transition System). A Labeled Transition System (LTS) is a tuple L= $(S$, init, Act,$\rightarrow)$ where $S$ is a finite set of states; init $\in S$ is an initial state; Act is a finite set of actions; and $\rightarrow \subseteq S \times$ Act $\times S$ is a labeled transition relation.

For simplicity, we write $s \stackrel{\alpha}{\rightarrow} s^{\prime}$ to denote $\left(s, \alpha, s^{\prime}\right) \in \rightarrow$. The set of enabled actions at $s$ is $\operatorname{enabled}(s)=\left\{\alpha \in\right.$ Act $\left.\mid \exists s^{\prime} \in S . s \stackrel{\alpha}{\rightarrow} s^{\prime}\right\}$. A path $\pi$ of $L$ is a sequence of alternating states and actions, starting and ending with states $\pi=\left\langle s_{0}, \alpha_{1}, s_{1}, \alpha_{2}, \cdots\right\rangle$ such that $s_{0}=$ init and $s_{i} \stackrel{\alpha_{i+1}}{\rightarrow} s_{i+1}$ for all $i$. If $\pi$ is finite, then $|\pi|$ denotes the number of transitions in $\pi$. A path can also be infinite, 


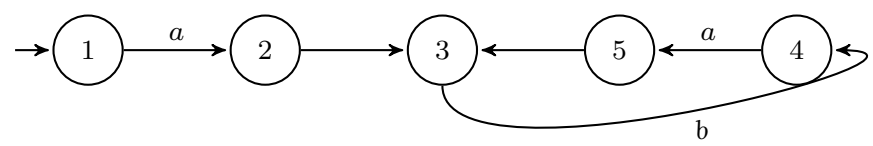

Figure 2.2: An LTS example

i.e., containing infinite number of actions. Since the number of states are finite, infinite paths are paths containing loops. The set of all possible paths for $L$ is written as paths $(L)$.

A transition label can be either a visible action or an invisible one. Given an LTS $L$, the set of visible actions in $L$ is denoted by $v i s_{L}$ and the set of invisible actions is denoted by $i n v i s_{L}$. A $\tau$-transition is a transition labeled with an invisible action. A state $s^{\prime}$ is reachable from state $s$ if there exists a path that starts from $s$ and ends with $s^{\prime}$, denoted by $s \stackrel{*}{\Rightarrow} s^{\prime}$. The set of $\tau$-successors is $\tau(s)=\left\{s^{\prime} \in S \mid s \stackrel{\alpha}{\rightarrow} s^{\prime} \wedge \alpha \in\right.$ invis $\left._{L}\right\}$. The set of states reachable from $s$ by performing zero or more $\tau$ transitions, denoted as $\tau^{*}(s)$, can be obtained by repeatedly computing the $\tau$-successors starting from $s$ until a fixed point is reached. We write $s \stackrel{\tau^{*}}{\rightarrow} s^{\prime}$ iff $s^{\prime}$ is reachable from $s$ via only $\tau$-transitions, i.e., there exists a path $\left\langle s_{0}, \alpha_{1}, s_{1}, \alpha_{2}, \cdots, s_{n}\right\rangle$ such that $s_{0}=s, s_{n}=s^{\prime}$ and $s_{i} \stackrel{\alpha_{i+1}}{\rightarrow} s_{i+1} \wedge \alpha_{i+1} \in$ invis $_{L}$ for all $i$. Given a path $\pi$, we can obtain a sequence of visible actions by omitting states and invisible actions. The sequence, denoted as $\operatorname{trace}(\pi)$, is a trace of $L$. The set of all traces of $L$, is written as $\operatorname{traces}(L)=\{\operatorname{trace}(\pi) \mid \pi \in \operatorname{paths}(L)\}$.

LTSs can often be shown graphically, e.g., Fig. 2.2 shows an example LTS, where invisible transition labels are omitted for simplicity. We define the refinement relation between two LTSs, usually called trace refinement, as follows.

Definition 6 (Refinement). Let $L_{1}$ and $L_{2}$ be two LTSs. $L_{1}$ refines $L_{2}$, written as $L_{1} \sqsupseteq_{T} L_{2}$ iff 
$\operatorname{traces}\left(L_{1}\right) \subseteq \operatorname{traces}\left(L_{2}\right)$.

In [15], we have shown that if $L_{i m p l}$ is an implementation LTS and $L_{\text {spec }}$ is the LTS of the linearizable specification, then $L_{\text {impl }}$ is linearizable if and only if $L_{i m p l} \sqsupseteq_{T} L_{\text {spec }}$.

Given two labeled transition systems $M_{\text {spec }}$ and $M_{i m p l}$ representing specification and implementation LTS respectively, we say that $M_{\text {impl }}$ refines $M_{\text {spec }}$ if and only if the set of execution traces in $M_{i m p l}$ is a subset of the execution traces in $M_{\text {spec }}$. So, the idea in refinement checking is to establish the (weak) simulation relationship between the specification model and the implementation model. The main approach is to perform the exhaustive search for the state space that is build of combined the specification-implementation. We compare every reachable state of the implementation with that of the specification reachable via same trace. If this condition suffices, we say that the $M_{\text {spec }}$ refines $M_{i m p l}$, otherwise we get a counterexample which violates the check.

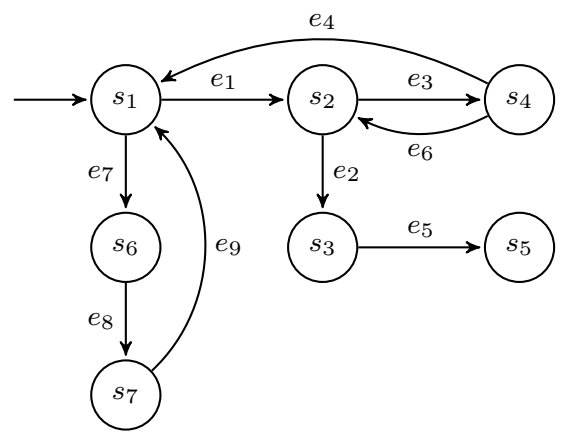

Figure 2.3: Specification model

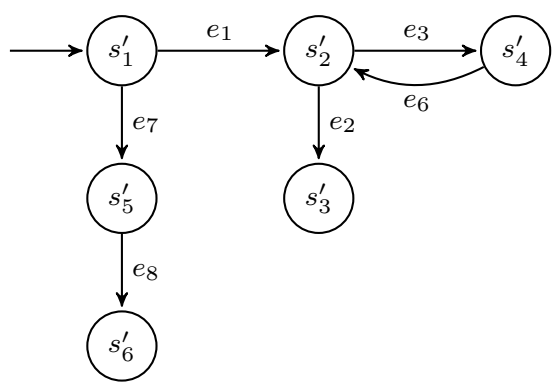

Figure 2.4: Implementation model

We start by presenting an example that illustrates the refinement checking. Fig. 2.3 shows the specification model and Fig. 2.4 shows the implementation model. Starting from the initial state $s_{1}^{\prime}$ in the implementation model, we check the existence of a state with an enabled event $e_{1}$ from 
the $s_{1}$ in the specification model. As, the Spec LTS also has the same transition from state $s_{1}$, we continue the search by grabbing the next state. We can use Breadth-First Search (BFS) or DepthFirst Search (DFS) to search the children. Considering a LTS with visible events $e$ and invisible events $\tau$, formally, we can grab the children of a state as follows:

1. if $i m p l \stackrel{\tau}{\rightarrow} i m p l^{\prime}$, where $\tau$ is an internal event, then spec $^{\prime}=$ spec;

2. if $i m p l \stackrel{e}{\rightarrow} i m p l^{\prime}$, where $e$ is a method call event, then $s p e c \stackrel{e}{\rightarrow} \operatorname{spec}^{\prime}$;

We continue the check till all states of the implementation model is exhausted. In the given example, all the traces in implementation model shown in Fig. 2.4 are contained within the specification model shown in Fig. 2.3, meaning implementation refines specification.

\subsection{Realization of a Model}

We use a tool called PAT to model spec and impl. It is a framework that supports the specification and verification of concurrent models. We model concurrent systems using a process algebra that is a variant of CSP. A LTS generated describes the behavior of the model. Basically CSP syntax is used in our model with the extension of shared variable. Table 2.1 shows the process definitions used in our model. Here CSP is extended such that shared variables are modeled alongside the processes to implement the different behaviors of the models.

Refinement checking is the method to verify if the behaviors of implementation follow the specification. In PAT, an assertion is specified to check the behaviors of two processes. Consider the two 
Table 2.1: Process Definitions

\begin{tabular}{|l|l|}
\hline Process & Definition \\
\hline \hline$P()=$ Stop & $\begin{array}{l}\text { A process that communicates nothing. } \\
\text { It is also called the deadlock process. }\end{array}$ \\
\hline$P()=$ Skip & Terminates an event. Skip is termination process. \\
\hline$P()=$ event $\{$ assignments $\} \rightarrow P()$ & $\begin{array}{l}\text { Describes that } \text { event is performed first and then it behaves } \\
\text { like a process } P() .\end{array}$ \\
\hline$P()=P_{1}() ; P_{2}() ;$ & $\begin{array}{l}\text { It is the sequential composition of two processes } P_{1} \text { and } P_{2} \\
P_{1}() \text { is executed first and } P_{2}() \text { is executed after that. }\end{array}$ \\
\hline$P()=P_{1}()|| \mid P_{2}()$ & $\begin{array}{l}\text { It is the interleaved composition of the two processes } \\
P_{1}() \text { and } P_{2}() .\end{array}$ \\
\hline
\end{tabular}

processes $P()$ and $Q()$. The statement \#assert $P()$ refines $Q()$ checks if there is a refinement relationship between these two processes $P()$ and $Q()$. The reachability analysis is conducted by exploring the product state transition of $P()$ and $Q()$, wherein the state that violates the refinement relationship is searched.

\subsection{Related Work}

In the literature, although there exists a large body of work on formally verifying linearizability in models of data structure implementations, none of them can verify quasi linearizability. For example, Liu et al. $[15,14]$ use a process algebra based tool to verify that an implementation model refines a specification model - the refinement relation implies linearizability. Vechev et al. [24] use the SPIN model checker to verify linearizability in a Promela model. Cerný et al. [5] use automated abstractions together with model checking to verify linearizability properties. There also exists some work on proving linearizability by constructing mechanical proofs, often with 
significant manual intervention (e.g., [22, 23]).

There are also runtime verification algorithms such as Line-Up [4], which can directly check the actual source code implementation but for violations on bounded executions and deterministic linearizability. However, quasi linearizable data structures are inherently nondeterministic. For example, the deq operation in a quasi queue implementation may choose to return any of the first $k$ items in a queue. To the best of our knowledge, no existing method can directly verify quasi linearizability for execution traces of unbounded length.

Besides (quasi) linearizability, there also exist many other consistency conditions for concurrent computations, including sequential consistency [13], quiescent consistency [2], and eventual consistency [25]. Some of these consistency conditions in principle may be used for checking the correctness of data structure implementations, although so far, none of them is as widely used as (quasi) linearizability. These consistency conditions do not involve quantitative aspects of the properties. We believe that it is possible to extend our refinement algorithm to verify some of these properties. However, we leave it for future work.

Outside the domain of concurrent data structures, serializability and atomicity are two popular correctness properties for concurrent programs, especially at the application level. There exists a large body of work on both static and dynamic analysis for detecting violations of such properties (e.g., $[8,6]$ and $[27,7,18,26])$. These existing methods are different from ours because they are checking different properties. Although atomicity and serializability are fairly general correctness conditions, they have been applied mostly to the correctness of shared memory accesses at the load/store instruction level. Linearizability, in contrast, defines correctness condition at the method 
call level. Furthermore, existing methods for checking atomicity and serializability do not deal with the quantitative aspects of the properties.

\subsection{Summary}

This chapter presented a brief overview of linearizability and quasi linearizability in relation to refinement. The idea of model checking by building the labeled transition systems and refinement checking was introduced. The chapter also included the related work on formal verification of linearizable data structures. 


\section{Chapter 3}

\section{Verifying Quasi Linearizability via}

\section{Refinement Checking}

This chapter presents our two new approaches for verifying quasi linearizability. We first explore two possible paths that allow us to verify quasi linearizability. First, we present the detailed description of a manual verification approach and show its limitations. This motivates our work in next chapter, which is extending the standard refinement checking algorithm to build an automated quasi linearizable checker. 


\subsection{Two Approaches of Verification}

Our verification problem is defined as follows: Given an implementation model $M_{i m p l}$, a specification model $M_{s p e c}$, and a quasi factor $Q_{O}$, decide whether $M_{i m p l}$ is quasi linearizable with respect to $M_{\text {spec }}$ under the quasi factor $Q_{O}$.
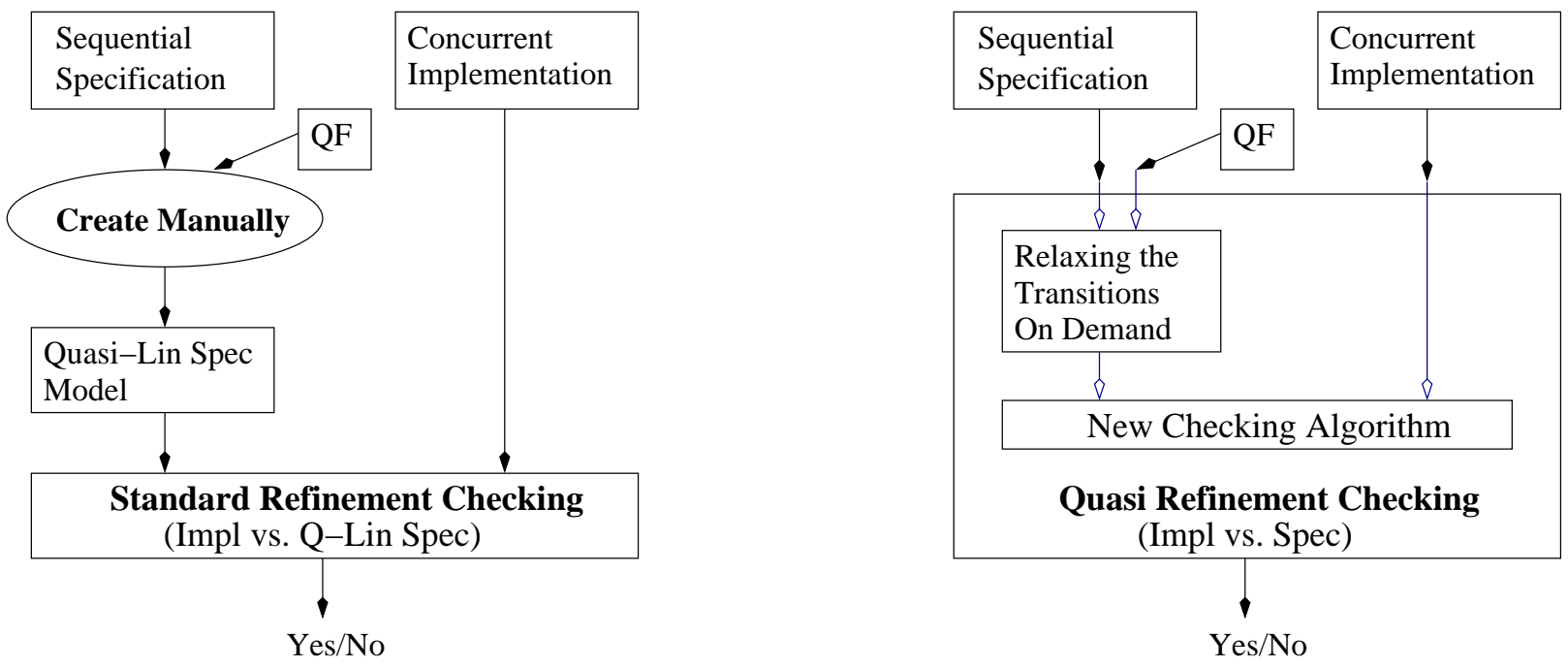

Figure 3.1: Verifying quasi linearizability: manual approach (left) and automated approach (right).

The straightforward approach for solving the problem is to leverage the procedure in Algorithm 3. However, since the procedure checks for standard refinement relation, not quasi refinement relation, the user has to manually construct a relaxed specification model, denoted $M_{\text {spec }}^{\prime}$, based on the given specification model $M_{\text {spec }}$ and the quasi factor $Q_{O}$. This so-called manual approach is illustrated by Fig. 3.1 (left). The relaxed specification model $M_{\text {spec }}^{\prime}$ must be able to produce all histories that can be produced by $M_{s p e c}$, as well as the new histories that are allowed under the relaxed consistency condition in Definition 4.

Unfortunately, there is no systematic method, or general guideline, on constructing such relaxed 
specification models. Each $M_{\text {spec }}^{\prime}$ may be different depending on the type of data structures to be checked. And there is significant amount of creativity required during the process, to make sure that the new specification model is both simple enough and permissive enough. For example, to verify that a $K$-segmented queue [1] is quasi linearizable, we can create a relaxed specification model whose dequeue method randomly removes one of the first $K$ data items from the otherwise standard FIFO queue. This new model $M_{\text {spec }}^{\prime}$ will be more complex than $M_{\text {spec }}$, but can still be significantly simpler than the full-fledged implementation model $M_{i m p l}$, which requires the use of a complex segmented linked list.

Since the focus of this thesis is on designing a fully automated verification method, we shall briefly illustrate the manual approach in this chapter, and then focus on developing an automated approach in the subsequent chapter.

Our automated approach is shown in Fig. 3.1 (right). It is based on designing a new refinement checking algorithm that, in contrast to Algorithm 3, can directly check a relaxed version of the standard refinement relation between $M_{i m p l}$ and $M_{\text {spec }}$. Therefore, the user does not need to manually construct the relaxed specification model $M_{\text {spec }}^{\prime \prime}$ Instead, inside the new refinement checking procedure, we systematically extend states and transitions of the specification model $M_{\text {spec }}$ so that the new states and transitions as required by $M_{\text {spec }}^{\prime}$ are added on the fly. This would lead to the inclusion of a bounded degree of out-of-order execution on the relevant subset of operations as defined by the quasi factor $Q_{O}$. A main advantage of our new method is that the procedure is fully automated, thereby avoiding the user intervention, as well as the potential errors that may be introduced during the user's manual modeling process. Furthermore, by exploring the 
relaxed transitions on a need-to basis, rather than upfront as in the manual approach, we can reduce the number of states that need to be checked.

\subsection{The Manual Verification Approach}

In this section, we will briefly describe the manual approach and then focus on presenting the automated approach in the subsequent chapter. Although we do not intend to promote the manual approach - since it is labor-intensive and error prune - this section will illustrate the intuitions behind our fully automated verification method.

Given the specification model $M_{\text {spec }}$ and the quasi factor $Q_{O}$, we show how to manually construct the relaxed specification model $M_{\text {spec }}^{\prime}$ in this section. We use the standard FIFO queue and two versions of quasi linearizable queues as examples. The construction needs to be tailored case by case for the different types of data structures.

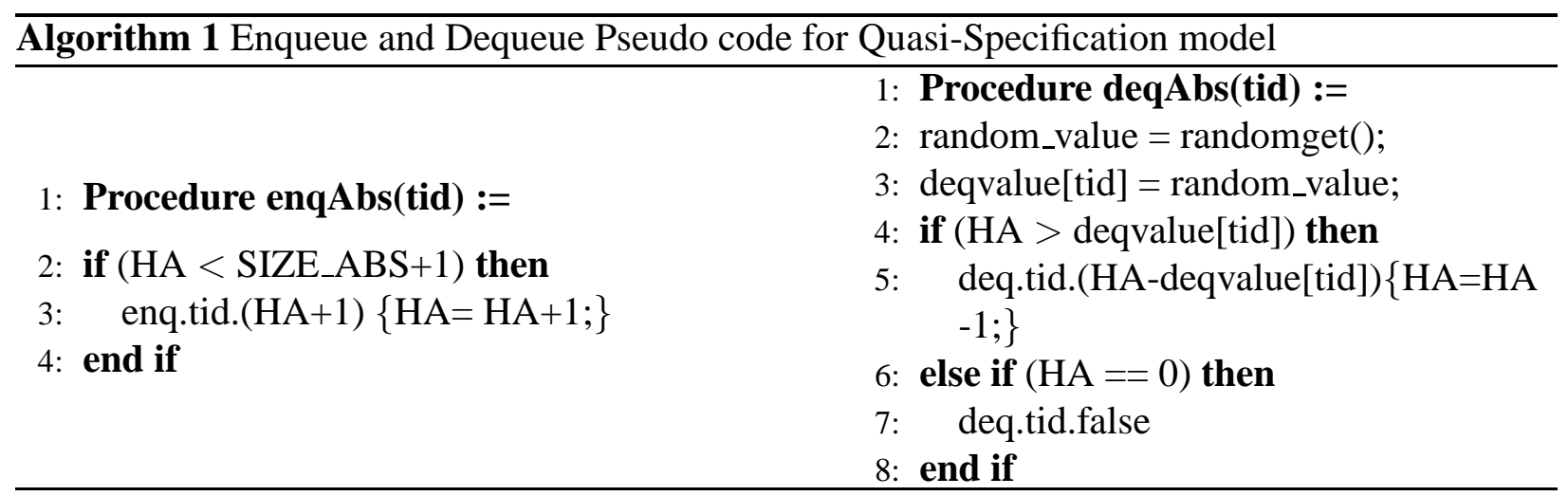




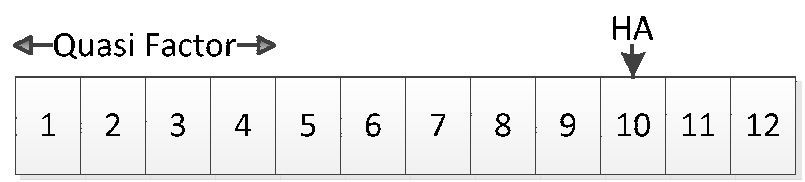

Quasi-Abstract Specification

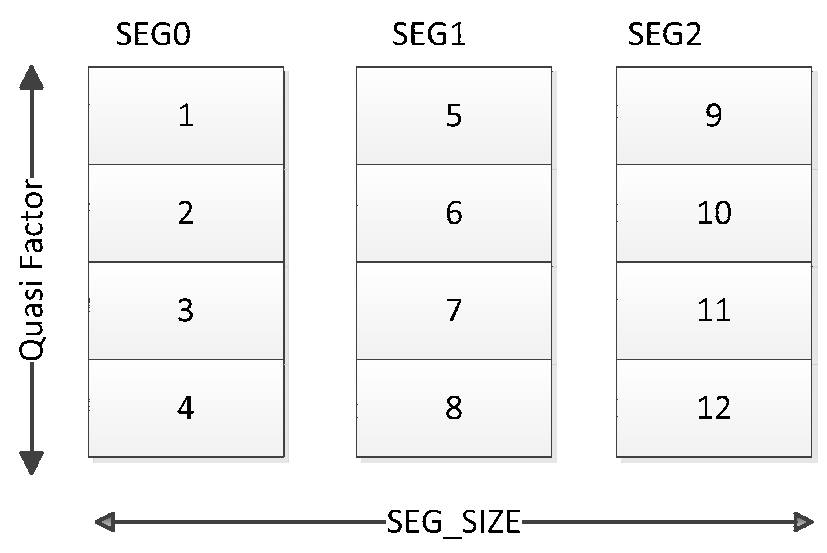

Quasi-Implementation

Figure 3.2: Implementations of a 4-quasi queue

\subsubsection{Specification Model $M_{\text {spec }}$}

The standard FIFO queue with a bounded capacity can be implemented by using a linked list, where deq operation removes a data item at one end of the list called the head node, and enq operation adds a data item at the other end of the list called the tail node. When the queue is full, enq does not have any impact. When the queue is empty, deq returns NULL. As an example, consider a sequence of four enqueue events enq (1), enq (2), enq (3), enq (4), the subsequent dequeue events would be deq.1, deq.2, deq.3, deq.4, which obey the FIFO semantics. This is illustrated by the first history H1-a in Fig. 3.3.

In the PAT model checking environment, the specification model $M_{\text {spec }}$ is written in a process 


$\begin{array}{llll}\text { H1-a } & \text { H1-b } & \text { H1-a } & \text { H1-b } \\ ------- & ------ & ------ & ------ \\ \operatorname{enq}(1) & \operatorname{enq}(1) & \operatorname{enq}(1) & \operatorname{enq}(1) \\ \operatorname{enq}(2) & \operatorname{enq}(2) & \operatorname{enq}(2) & \operatorname{enq}(2) \\ \operatorname{enq}(3) & \operatorname{enq}(3) & \operatorname{enq}(3) & \operatorname{enq}(3) \\ \operatorname{enq}(4) & \operatorname{enq}(4) & \operatorname{enq}(4) & \operatorname{enq}(4) \\ \operatorname{deq}()=1 & \operatorname{deq}()=1 & \operatorname{deq}()=2 & \operatorname{deq}()=2 \\ \operatorname{deq}()=2 & \operatorname{deq}()=2 & \operatorname{deq}()=1 & \operatorname{deq}()=1 \\ \operatorname{deq}()=3 & \operatorname{deq}()=4 & \operatorname{deq}()=3 & \operatorname{deq}()=4 \\ \operatorname{deq}()=4 & \operatorname{deq}()=3 & \operatorname{deq}()=4 & \operatorname{deq}()=3 \\ ------- & ------- & ------- & -------\end{array}$

Here, deq can be out-of-order by 1 . The first deq randomly returns a value from the set $\{1,2\}$ and the second deq returns the remaining one. Then the third deq randomly returns a value from the set $\{3,4\}$ and the forth deq returns the remaining one.

Figure 3.3: Valid histories of a 1-quasi linearizable queue

algebra language, named CSP\# [19]. Algorithm 1 shows the detailed explaination for creating an abstract model in the PAT tool environment.

\subsubsection{Implementation Model $M_{i m p l}$ :}

The bounded quasi linearizable queue can be implemented by using a segmented linked list. This is the original algorithm proposed by Afek et al. [1]. A segmented linked list is a linked list where each list node can hold $K$ data items, as opposed to a single data item in the standard linked list. As shown in Fig. 3.2 (lower half), these $K$ data items form a segment, in which the data slots are numbered as $1,2, \ldots, \mathrm{K}$. In general, the segment size needs to be set to $(Q F+1)$, where $Q F$ is the maximum number of out-of-order execution steps. The example in Fig. 3.2 has the quasi factor set to 3, meaning that a deq operation can be executed out of order by at most 3 steps. Consequently, the size of each segment is set to $(3+1)=4$. Since $Q_{\{\text {queue }\}}\left(D_{\text {enq }}\right)=0$, meaning that the eng 


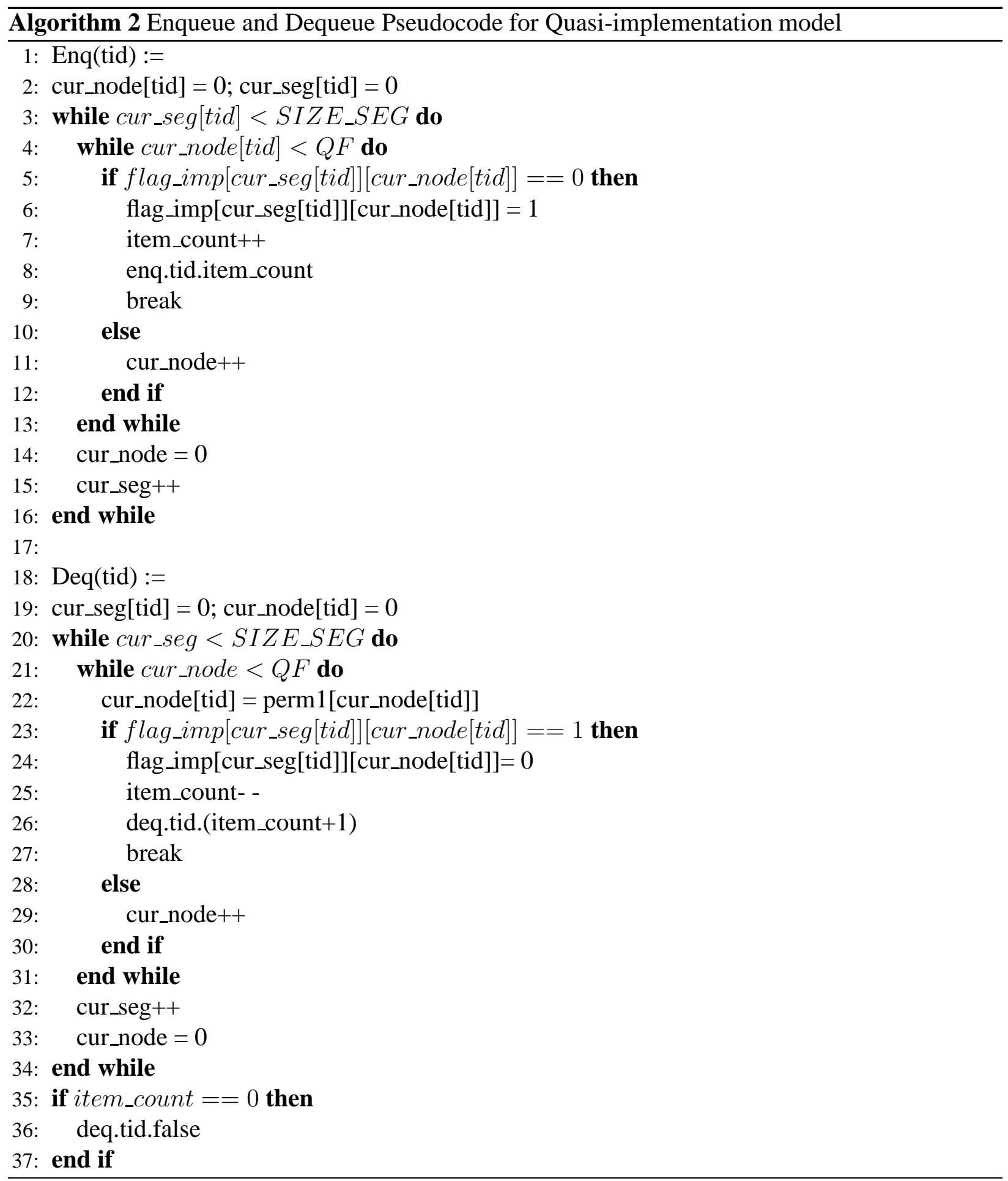


operations cannot be reordered, the data items are enqueued regularly in the empty slots of one segment, before the head points to the next segment. But for deq operations, we randomly remove one existing data item from the current segment. Algorithm 2 shows the detailed explaination for creating an implementation model in the PAT tool environment.

\subsubsection{Relaxed Specification Model $M_{\text {spec }}^{\prime}$ :}

Not all execution traces of $M_{i m p l}$ are traces of $M_{\text {spec }}$. In Fig. 3.3, histories other than H1-a are not linearizable. However, they are all quasi linearizable under the quasi factor 1 . They may be produced by a segmented queue where the segment size is $(1+1)=2$. To verify that $M_{i m p l}$ is quasi linearizable, we construct a new model $M_{\text {spec }}^{\prime}$, which includes not only all histories of $M_{\text {spec }}$ but also the histories that are allowed only under the relaxed consistency condition. In this example, we choose to construct the new model by slightly modifying the standard FIFO queue. This is illustrated in Fig. 3.2 (upper half), where the first $K$ data items are grouped into a cluster. Within the cluster, the deq operation may remove any of the $k$ data items based on randomization. Only after the first $k$ data items in the cluster are retrieved, will the deq move to the next $k$ data items (a new cluster). The external behavior of this model is expected to match that of the segmented queue in $M_{i m p l}$ : both are 1-quasi linearizable. 


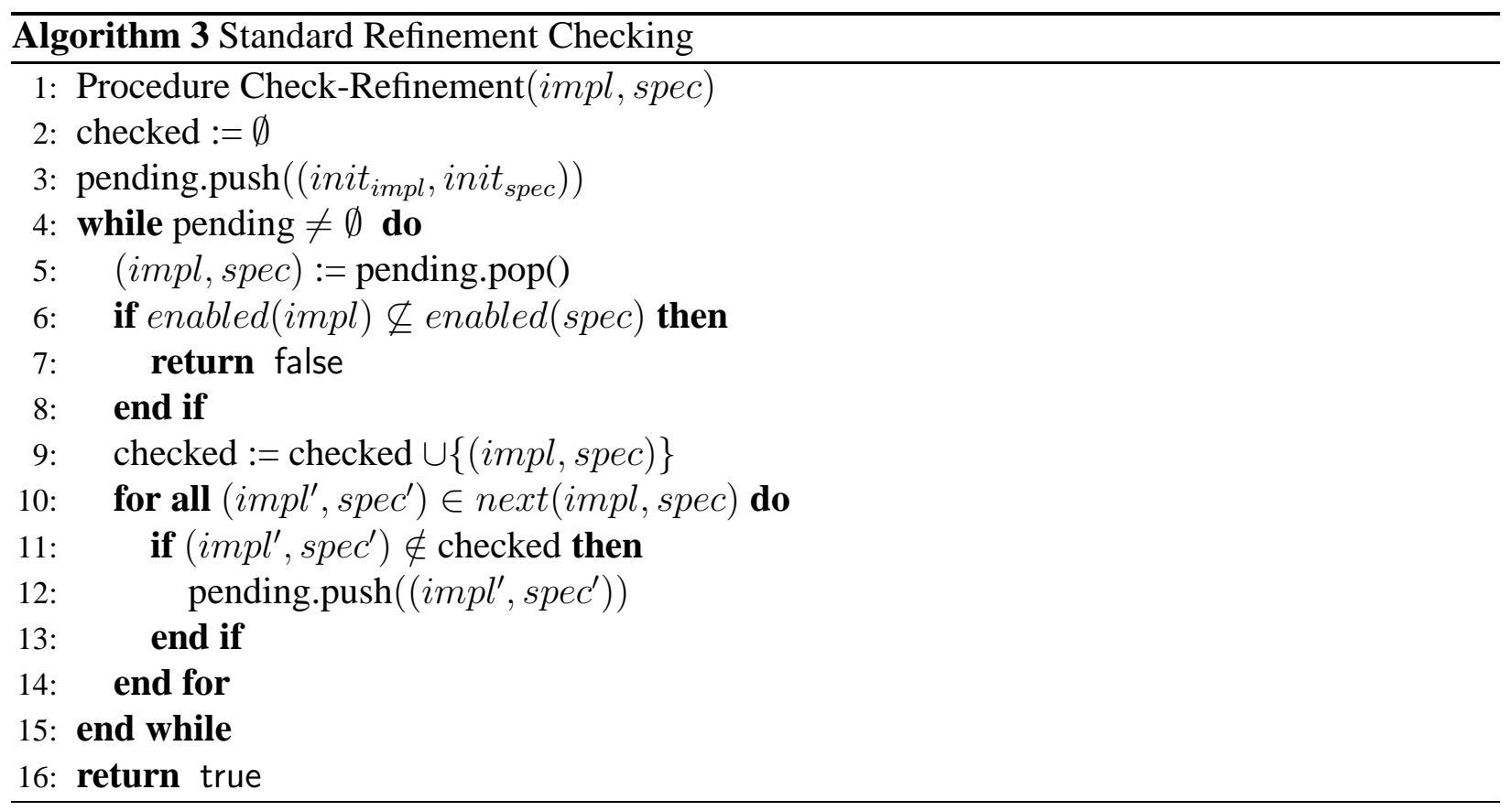

\subsubsection{Checking Refinement Relation:}

Once $M_{\text {spec }}^{\prime}$ is available, checking whether $M_{\text {impl }}$ refines $M_{\text {spec }}^{\prime}$ is straightforward by using Algorithm 3. Algorithm 3 shows the pseudo code of the refinement checking procedure in [15, 14]. Assume that $L_{i m p l}$ refines $M_{\text {spec }}$, then for each reachable transition in $M_{i m p l}$, denoted as $i m p l \stackrel{e}{\rightarrow}$ $i m p l^{\prime}$, there must exist a reachable transition in $L_{\text {spec }}$, denoted as $s p e c \stackrel{e}{\rightarrow} s p e c^{\prime}$. Therefore, the procedure starts with the pair of initial states of the two models, and repeatedly checks whether they have matching successor states. If the answer is no, the check at Lines 6-8 would fail, meaning that $L_{i m p l}$ is not linearizable. Otherwise, for each pair of immediate successor states $\left(i m p l^{\prime}, s p e c^{\prime}\right)$, we add the pair to the pending list. The entire procedure continues until either (1) a non-matching transition in $L_{i m p l}$ is found at Lines 6-8, or (2) all pairs of reachable states are checked, in which case $L_{i m p l}$ is proved to be linearizable. 
In Algorithm 3, the subroutine next $(\mathrm{impl}, \mathrm{spec})$ is crucially important. It takes the current states of $L_{i m p l}$ and $L_{\text {spec }}$ as input, and returns a set of state pairs of the form $\left(i m p l^{\prime}, s p e c^{\prime}\right)$. Here each pair $\left(i m p l^{\prime}, s p e c^{\prime}\right)$ is one of the immediate successor state pairs of $(i m p l, s p e c)$. They are defined as follows:

1. if $i m p l \stackrel{\tau}{\rightarrow} i m p l^{\prime}$, where $\tau$ is an internal event, then let $s p e c^{\prime}=$ spec;

2. if $i m p l \stackrel{e}{\rightarrow} i m p l^{\prime}$, where $e$ is a method call event, then $s p e c \stackrel{e}{\rightarrow} \operatorname{spec}^{\prime}$;

We have assumed, without loss of generality, that the specification model $L_{\text {spec }}$ is deterministic. If the original specification model is nondeterministic, we can always apply standard subset construction (of DFAs) to make it deterministic.

\subsection{Experimental Results}

For the segmented queue implementation [1], we have manually constructed $M_{s p e c}^{\prime}$ and checked the refinement relation in the PAT model checking environment. Our experimental results are summarized in Table 3.1. Column 1 shows the different quasi factors. Column 2 shows the number of segments - the capacity of the queue is $(Q F+1) \times S e g$. Column 3 shows the refinement checking time in seconds. Column 4 shows the total number of visited states during refinement checking. Column 5 shows the total number of state transitions activated during refinement checking. The experiments are conducted on a computer with an Intel Core-i7, $2.5 \mathrm{GHz}$ processor and 8 GB RAM running Ubuntu 10.04. 
Table 3.1: Experimental results for standard refinement checking.

\begin{tabular}{|c|c|c|c|c|}
\hline Quasi Factor & \#. Segment & Verification Time (s) & \#. Visited State & \#. Transition \\
\hline \hline 1 & 1 & 0.1 & 423 & 778 \\
\hline 1 & 2 & 0.1 & 2310 & 4458 \\
\hline 1 & 3 & 0.1 & 8002 & 15213 \\
\hline 1 & 4 & 0.4 & 22327 & 41660 \\
\hline 1 & 5 & 0.9 & 55173 & 101443 \\
\hline 1 & 6 & 2.0 & 126547 & 230259 \\
\hline 1 & 10 & 55.9 & 2488052 & 4421583 \\
\hline 1 & 15 & MOut & - & - \\
\hline \hline 2 & 1 & 0.6 & 26605 & 58281 \\
\hline 2 & 2 & 12.6 & 456397 & 970960 \\
\hline 2 & 3 & 130.7 & 4484213 & 8742485 \\
\hline 2 & 4 & MOut & - & - \\
\hline \hline 3 & 1 & 8.8 & 284484 & 638684 \\
\hline \hline 3 & 2 & MOut & - & - \\
\hline \hline 4 & 1 & 124.4 & 3432702 & 7906856 \\
\hline 4 & 2 & MOut & - & - \\
\hline
\end{tabular}

MOut means memory-out.

The experimental results in Table 3.1 show an exponential increase in the verification time when we increase the size of the queue or the quasi factor. For size $=1$, verification completes as soon as 0.1 seconds. When it is increased to 2 , it takes around 0.6 seconds to check if the implementation model refines specification or not. This is inevitable since the size of the state space grows exponentially as the size increases. Furthermore, as the quasi factor increases, the verification time is also significantly increased. We can see that for quasi factor as small as 1 , it takes only 0.1 seconds to complete the verification. But, when quasi factor is increased to 2 and 3 subsequently, there is tremendous increase in the visited states count and hence the verification time. Fig. 3.4 shows the relation of quasi factor and the verification time. The horizontal axis represents the quasi factor. Primary vertical axis represents the verification time for the corresponding quasi factor and the segment size. And, the secondary vertical axis represents the segment size of the queue. 


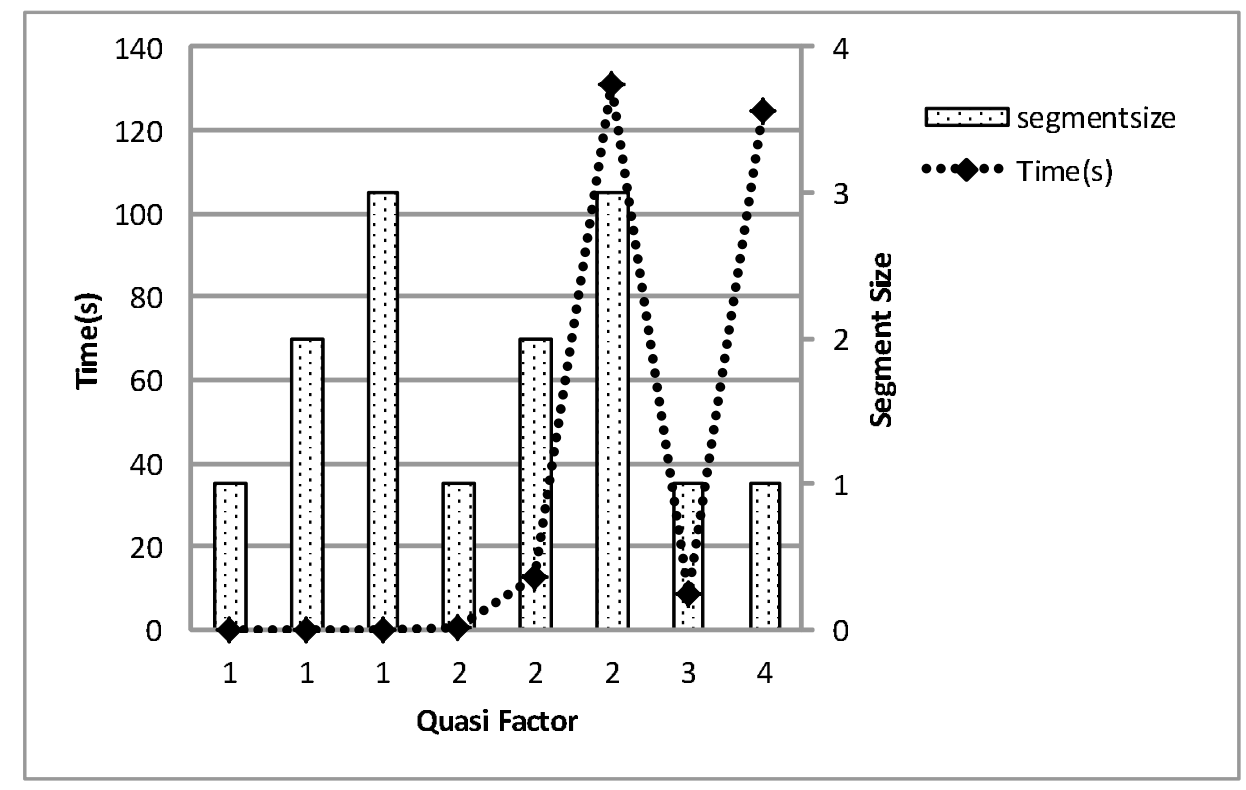

Figure 3.4: Graph showing the linearizability checking time for different QFs and segment sizes of a queue

\subsection{Limitations of the Manual Approach}

The proposed method is limited by the availability of the specification model in order to compare with the implementation models. One needs to know the possible traces for a model in order to check the correctness of the implementation model. So, this method requires the user to manually construct $M_{\text {spec }}^{\prime}$, which is a severe limitation because it is often labor intensive and error prone. For example, consider the seemingly simple random dequeued model in Fig. 3.2. A subtle error would be introduced if we do not use the cluster to restrict the set of data items that can be removed by deq operation. Assume that deq always returns one of the first $k$ data items in the current queue. Although it appears to be correct, such implementation will not be $k$-quasi linearizable, because it is possible for some data item to be over-taken indefinitely. For example, if every time deq chooses the second data item in the list, we will have the following deq sequence: deq.2, deq.3, 
deq. $4, \ldots$, deq. 1 , where the dequeue of value 1 can be delayed by an arbitrarily long time. This is no longer a 1-quasi linearizable queue. In other words, if the user construct $M_{\text {spec }}^{\prime}$ incorrectly, the verification result becomes invalid.

Therefore, we need to design a fully automated method to directly verify quasi linearizability of $M_{i m p l}$ against $M_{\text {spec }}$ under the given quasi factor $Q F$.

\subsection{Summary}

In this chapter, we proposed a model checking approach to verify the quasi linearizability of a concurrent data structure. Since, this technique is labor intensive and error prone, we proceed with the fully automated approach for checking the Quasi Linearizability in the next chapter. 


\section{Chapter 4}

\section{New Algorithm for Checking the Quasi}

\section{Refinement Relation}

In this chapter, we present our automated method for verifying Quasi Linearizability. We shall start with the standard refinement checking procedure in Algorithm 3 and extend it to directly check a relaxed version of the refinement relation between $M_{i m p l}$ and $M_{\text {spec }}$ under a given quasi factor. The idea is to establish the simulation relationship from specification to implementation while allowing relaxation of the specification. This not only reduces the chances of committing error during specification construction phase, but also automates the tool which can verify the correctness of concurrent data structures. 


\subsection{Motivation}

The manual verification approach presented in Chapter 3 not only can check the validity of the concurrent data structures, but also can generate the counterexample if the implementation model does not refine the specification. However, this is obtained at the cost of extensive manual effort used to model the quasi linearizable specification, which should contain all the possible traces that a relaxed linearizable implementation could have. Also, the possibility of introducing errors while writing the specification model is high. Therefore, we propose a new and fully automated method which relaxes the specification of a model first and then conduct refinement checking to ascertain the correctness of the implementation model.

\subsection{Linearizability Checking via Quasi Refinement}

The new procedure, shown in Algorithm 4, is different from Algorithm 3 as follows:

1. We customize pending to make the state exploration follow a breadth-first search (BFS). In Algorithm 3, it can be either BFS or DFS based on whether pending is a queue or stack.

2. We replace enabled(spec) with enabled_relaxed(spec, $Q F)$. It will return not only the events enabled at current spec state in $M_{\text {spec }}$, but also the additional events allowed under the relaxed consistency condition.

3. We replace next(impl,spec) with next_relaxed(impl,spec, $Q F)$. It will return not only the successor state pairs in the original models, but also the additional pairs allowed under the 


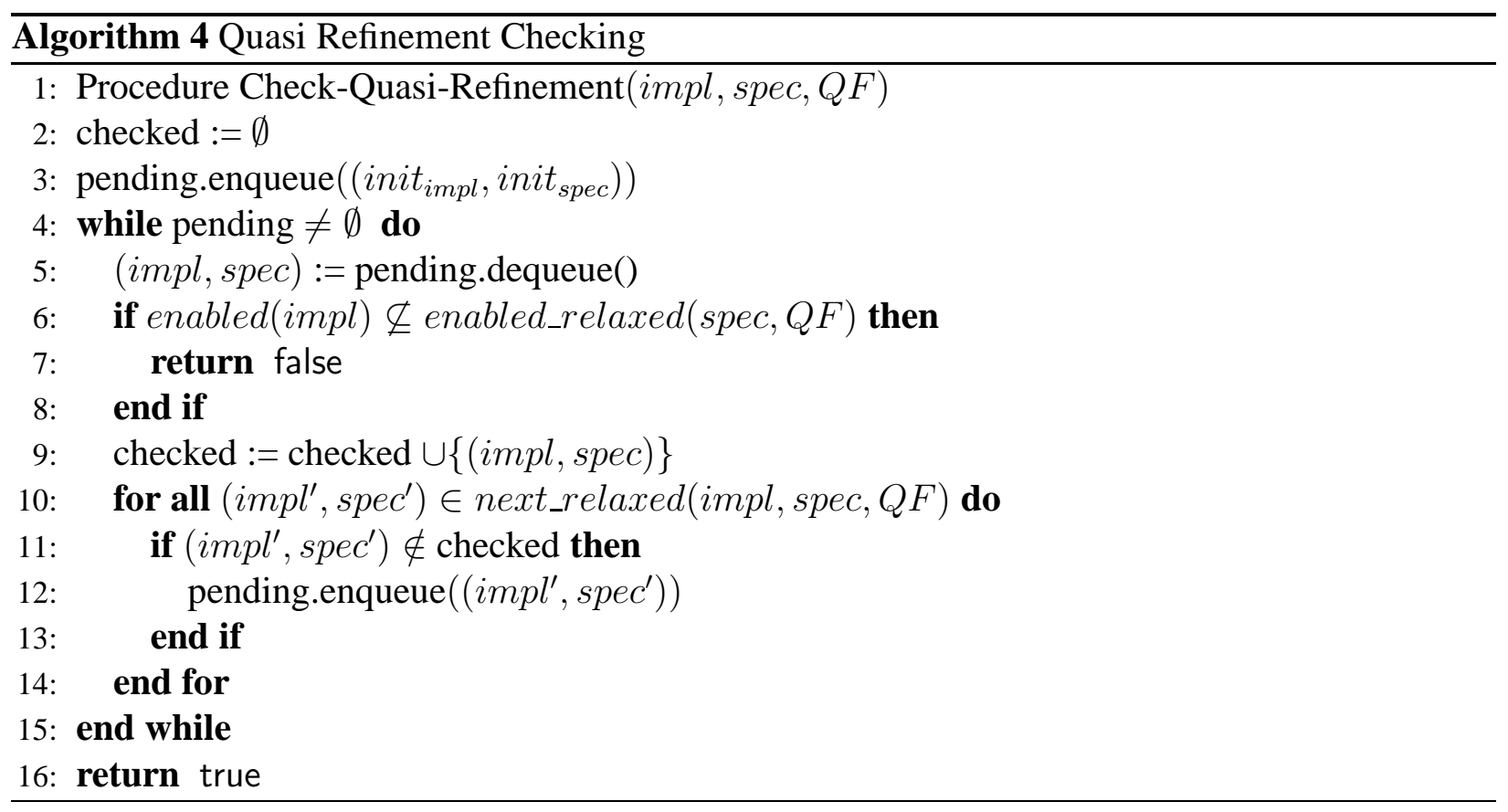

relaxed consistency condition.

Conceptually, it is equivalent to first constructing a relaxed specification model $M_{\text {spec }}^{\prime}$ from $\left(M_{\text {spec }}, Q F\right)$ and then computing the enabled(spec) and next(impl,spec) on this new model. However, in this case, we are constructing $M_{\text {spec }}^{\prime}$ automatically, without the user's intervention. Furthermore, the additional states and edges that need to be added to $M_{\text {spec }}^{\prime}$ are processed incrementally, on a need-to basis.

At the high level, the new procedure performs a BFS exploration for the state pair $(\mathrm{impl}, \mathrm{spec})$, where impl is the state of implementation and spec is a state of specification. The initial implementation and specification events are enqueued into pending and each time we go through the while-loop, we dequeue from pending a state pair, and check if all events enabled at state $i m p l$ match with some events enabled at state spec under the relaxed consistency condition (line 6). 
If there is any mismatch, the check fails and we can return a counterexample showing how the violation happens. Otherwise, we continue until pending is empty. Lines 10-14 explore the new successor state pairs, by invoking next_relaxed and add to pending if they have not been checked.

Subroutine enabled_relaxed(spec,QF): It takes the current state spec of model $M_{\text {spec }}$, along with the quasi factor $Q F$, and generates all events that are enabled at state spec.

Consider the graph in Fig. 4.1 as $M_{\text {spec }}$. Without relaxation, enabled $\left(s_{1}\right)=\left\{e_{1}\right\}$. This is equivalent to enabled_relaxed $\left(s_{1}, 0\right)$. However, when $Q F=1$, according to the dotted edges in Fig. 4.2, the set enabled_relaxed $\left(s_{1}, 1\right)=\left\{e_{1}, e_{2}, e_{3}\right\}$.

The reason why $e_{2}$ and $e_{3}$ become enabled is as follows: before relaxation, starting at state $s_{1}$, there are two length-3 $(2 Q F+1)$ event sequences $\sigma_{1}=e_{1}, e_{2}, e_{5}$ and $\sigma_{2}=e_{1}, e_{3}, e_{4}$. When $Q F=1$, it means an event can be out-of-order by at most 1 step. Therefore, the possilbe valid permutations of $\sigma_{1}$ is $\pi_{1}=e_{2}, e_{1}, e_{5}$ and $\pi_{2}=e_{1}, e_{5}, e_{2}$, and the possible valid permutations of $\sigma_{2}$ is $\pi_{3}=e_{3}, e_{1}, e_{4}$ and $\pi_{4}=e_{1}, e_{4}, e_{3}$ for $Q F=1$. In other words, at state $s_{1}$, events $e_{2}, e_{3}$ can also be executed. We will discuss the generation of valid permutation sequences in Section 4.3.

Subroutine next_relaxed $(i m p l, s p e c, Q F)$ : It takes the current state $i m p l$ of $M_{i m p l}$ and the current state spec of $M_{\text {spec }}$ as input, and returns a set of state pairs of the form $\left(i m p l^{\prime}\right.$, spec $\left.^{\prime}\right)$. Similar to the definition of next $(i m p l$, spec $)$ in Section ??, we define each pair $\left(i m p l^{\prime}\right.$, spec $\left.c^{\prime}\right)$ as follows:

1. if $i m p l \stackrel{\tau}{\rightarrow} i m p l^{\prime}$, where $\tau$ is an internal event, then let $\operatorname{spec}^{\prime}=$ spec; 
2. if $i m p l \stackrel{e}{\rightarrow} i m p l^{\prime}$, where $e$ is a method call event, then $s p e c \stackrel{e}{\rightarrow}$ spec $^{\prime}$ where event $e \in$ enabled_relaxed $($ spec, $Q F)$ is enabled at spec after relaxation.

For example, when spec $=s_{1}$ in Fig. 4.1, and the quasi factor is set to $1-$ meaning that the event at state $s_{1}$ can be out-of-order by at most one step - the procedure next_relaxed(impl, $\left.s_{1}, 1\right)$ would return not only $\left(i m p l^{\prime}, s_{2}\right)$, but also $\left(i m p l^{\prime}, s_{6}\right)$ and $\left(i m p l^{\prime}, s_{9}\right)$, as indicated by the dotted edges in Fig. 4.2. The detailed algorithm for generation of the relaxed next states in specification is described in Section 4.3.

\subsection{Generation of Relaxed Specification}

In this subsection, we show how to relax the specification $M_{\text {spec }}$ by adding new states and transitions - those that are allowed under the condition of quasi linearizability - to form a new specification model. Notice that we accomplish this automatically, and incrementally, on a need-to basis.

For each state spec in $M_{\text {spec }}$, we compute all the event sequences starting at spec with the length $(2 Q F+1)$. These event sequences can be computed by using a simple graph traversal algorithm, e.g., a breadth first search.

Fig. 4.1 shows an example for the computation of these event sequences. The specification model $M_{\text {spec }}$ has the following set of states $\left\{s_{1}, s_{2}, s_{3}, s_{4}, s_{5}\right\}$. Suppose that the current state is $s_{1}$ (in step 0 ), then the current frontier state set is $\left\{s_{1}\right\}$, and the current event sequence is $\left\langle s_{1}\right\rangle$. The 


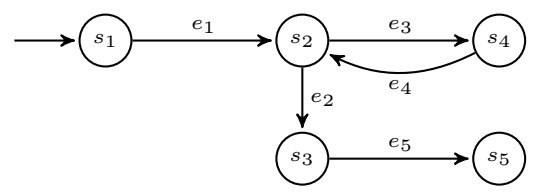

Figure 4.1: Specification model before adding relaxed transitions for state $s_{1}$.

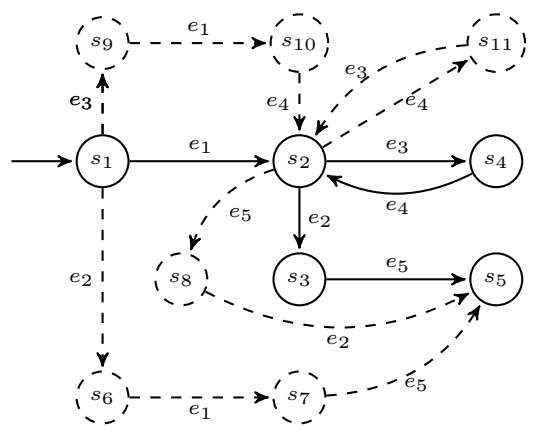

Figure 4.2: Specification model after adding relaxed edges for state $s_{1}$ and quasi factor 1 .

Table 4.1: Specification sequence generation at state $s_{1}$

\begin{tabular}{|l|l|l|}
\hline BFS Steps & (Frontier) & EventSequences \\
\hline step 0 & $\left\{s_{1}\right\}$ & $\left\langle s_{1}\right\rangle$ \\
step 1 & $\left\{s_{2}\right\}$ & $\left\langle s_{1} \stackrel{e_{1}}{\rightarrow} s_{2}\right\rangle$ \\
step 2 & $\left\{s_{3}, s_{4}\right\}$ & $\left\langle s_{1} \stackrel{e_{1}}{\rightarrow} s_{2} \stackrel{e_{2}}{\rightarrow} s_{3}\right\rangle\left\langle s_{1} \stackrel{e_{1}}{\rightarrow} s_{2} \stackrel{e_{3}}{\rightarrow} s_{4}\right\rangle$ \\
step 3 & $\left\{s_{5}, s_{2}\right\}$ & $\left\langle s_{1} \stackrel{e_{1}}{\rightarrow} s_{2} \stackrel{e_{2}}{\rightarrow} s_{3} \stackrel{e_{5}}{\rightarrow} s_{5}\right\rangle\left\langle s_{1} \stackrel{e_{1}}{\rightarrow} s_{2} \stackrel{e_{3}}{\rightarrow} s_{4} \stackrel{e_{4}}{\rightarrow} s_{2}\right\rangle$ \\
\hline
\end{tabular}

results of each BFS step are shown in Table 4.1. In step 1 , the frontier state set is $\left\{s_{2}\right\}$, and the event sequence becomes $\left\langle s_{1} \stackrel{e_{1}}{\rightarrow} s_{2}\right\rangle$. In step 2 , the frontier state set is $\left\{s_{3}, s_{4}\right\}$, and the event sequence is split into two sequences. One is $\left\langle s_{1} \stackrel{e_{1}}{\rightarrow} s_{2} \stackrel{e_{2}}{\rightarrow} s_{3}\right\rangle$ and the other is $\left\langle s_{1} \stackrel{e_{1}}{\rightarrow} s_{2} \stackrel{e_{3}}{\rightarrow} s_{4}\right\rangle$. The traversal continues until the BFS depth reaches $(2 Q F+1)$.

After completing the $(2 Q F+1)$ steps of BFS starting at state $s p e c$, as above, we have to generate possible valid permutations first and then we will be able to evaluate the two subroutines: enabled_relaxed $($ spec, $Q F)$ and next_relaxed $(\mathrm{impl}$, spec, $Q F)$.

We transform the original specification model in Fig. 4.1 to the relaxed specification model in Fig. 4.2 for $Q F=1$. The dotted states and edges are newly added to reflect the relaxation. More specifically, for $Q F=1$, we will reach $(2 Q F+1)=3$ steps during the BFS. At step 3 , there are two existing sequences $\left\{e_{1}, e_{2}, e_{5}\right\}$ and $\left\{e_{1}, e_{3}, e_{4}\right\}$. For each existing sequence, we 
compute all possible valid permutation sequences. In this case, the valid permutation sequences are $\left\{e_{2}, e_{1}, e_{5}\right\},\left\{e_{1}, e_{5}, e_{2}\right\}$ and $\left\{e_{3}, e_{1}, e_{6}\right\},\left\{e_{1}, e_{3}, e_{6}\right\}$. For each newly generated permutation sequence, we add new edges and states to the specification model. From an initial state $s_{1}$, if we follow the new permutation $\left\{e_{2}, e_{1}, e_{5}\right\}$, as shown in Fig. 4.2, the transition $e_{2}$ will lead to newly formed pseudo state $s_{6}$, the transition $e_{1}$ will lead to $s_{7}$ from state $s_{6}$ and from this state it is reconnected back to the original state $s_{5}$ via transition $e_{5}$. Similarly, if we follow the new permutation $\left\{e_{3}, e_{1}, e_{4}\right\}$, the transition $e_{3}$ will lead to newly formed pseudo state $s_{9}$, the transition $e_{1}$ will lead to $s_{10}$ from state $s_{9}$ and from this state it is reconnected back to state $s_{2}$ via transition $e_{4}$. We continue this process of state expansion for all the valid permutation sequences. This relaxation process needs to be conducted by using every existing state of $M_{\text {spec }}$ as the starting point (for BFS up to $2 Q F+1$ steps) and then adding the new states and edges. Note that this process is conducted on the fly.

Algorithm 5 explains the high level pseudo-code for expanding the state space for the current specification state under the check. Let $S E Q=\left\{s e q_{1}, s e q_{2}, \ldots, s e q_{k}\right\}$ be the sequences which are reachable from the state $s_{0}$ in $M_{\text {spec }}$ such that each sequence has less than or equal to $2 Q F+1$ events. Each sequence $s e q \in S E Q$ calls a genValidPermut(seq, $Q F$ ) (line 4) to generate all the possible valid permutation paths for that trace. A new state is formed with a new transition for each event in the permuted sequences, hence allowing the relaxed refinement checking of the implementation trace.

The valid permutations for a given sequence is generated using an Algorithm 6 which is based on the cost associated with the event. Initially, for each events $e_{i}$ where $1 \leq i<n$ associated with the 


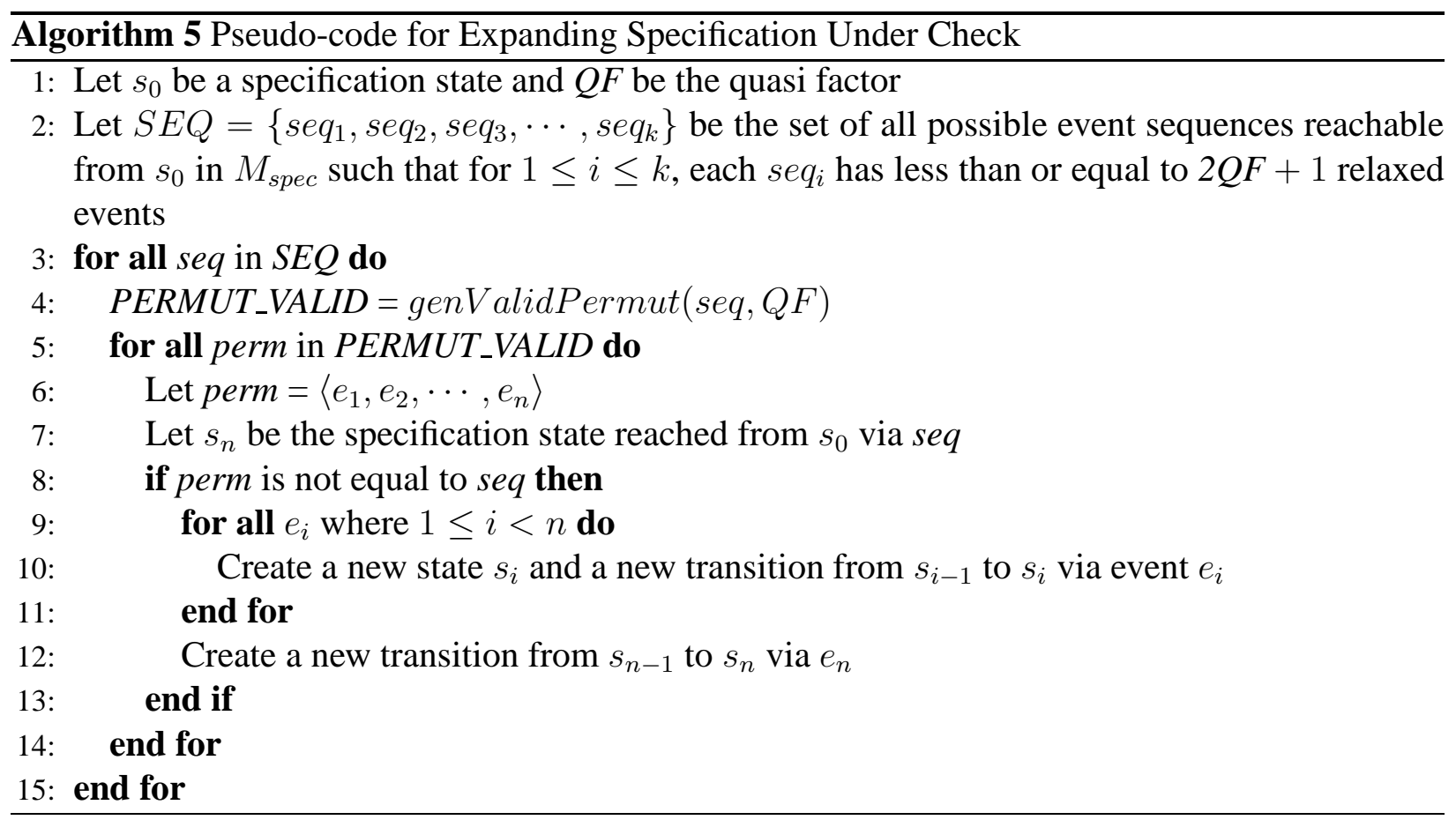

seq, the cost is initialized to $Q F$ (line 2). We generate all possible permutations and update cost with respect to the relative ordering of the events for each reshuffled sequences. This cost attribute of an event stores the information on how many more steps an event may be postponed. Each time an event is postponed, the cost associated with this event is decremented by 1 . On the contrary, the event can also be chosen upto $Q F$ steps ahead and for each step, the cost is increased by 1 . So, the cost attribute of the event that is allowed for relaxation is $2 Q F \leq \operatorname{cost} \leq 0$. We check the validity of each of these sequences using this cost attribute (line 8). Finally, only the valid permutations are appended in PERMUT_VALID after each check and once the check is completed for all permuted sequences, the function returns the valid traces.

Consider the event sequence $\left\{e_{1}, e_{2}, e_{5}\right\}$ from state $s_{1}$ be seq as shown in Fig. 4.1. If $Q F=1$, the cost for each of these events is initialized to 1 . We generate all possible permutations by reshuffling 


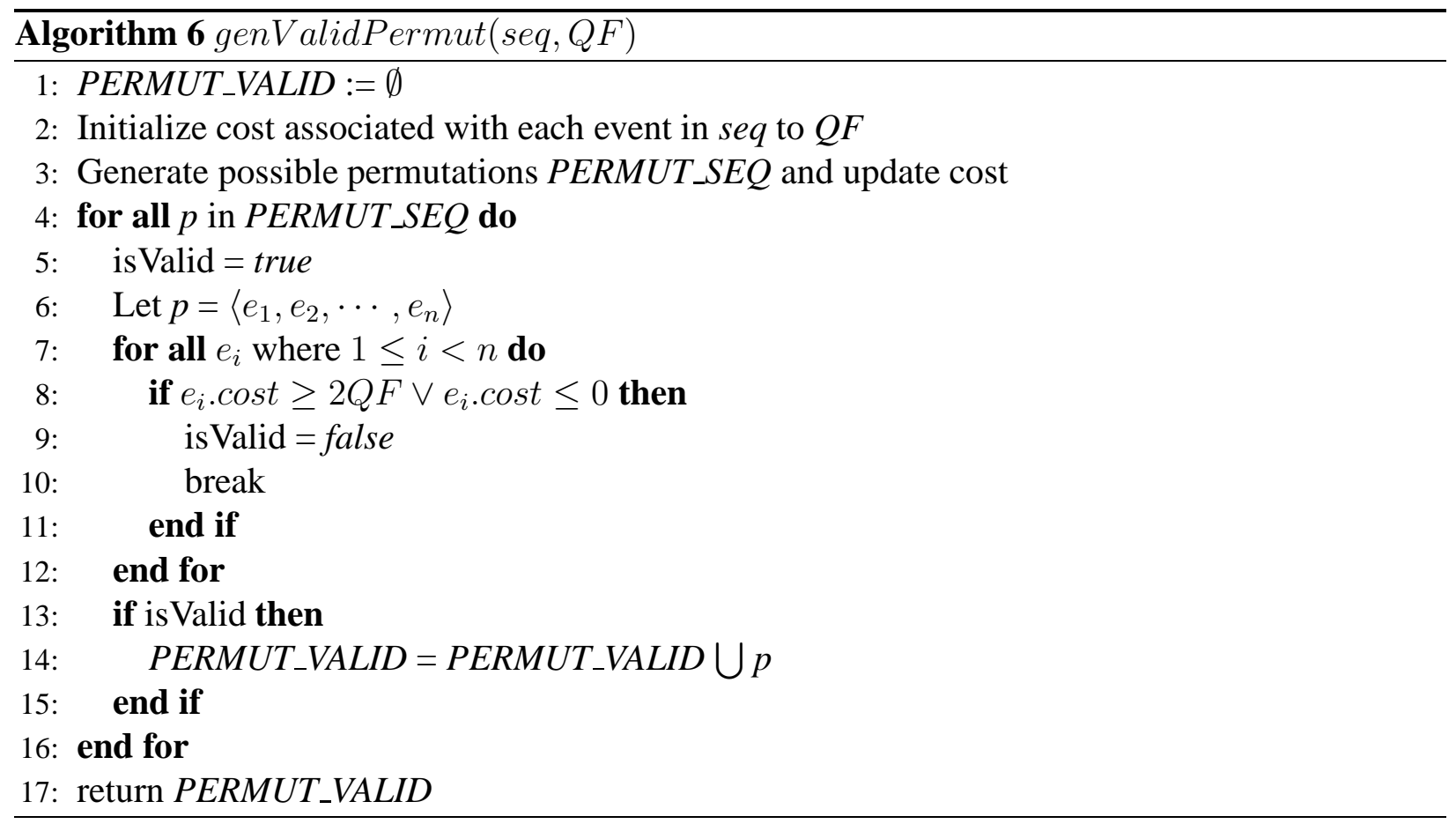

the events and updating the cost based on the relative positioning of the event with respect to the initial sequence. There are as many as 6 possible permutations including the original sequence in this case. If we consider reordering be the sequence $\left\{e_{2}, e_{1}, e_{5}\right\}$, then the cost associated with event $e_{2}$ is 2 as it is chosen one step earlier. For the event $e_{1}$, it is postponed for one step meaning its cost is decreased by 1 which makes the cost associated with it be 0 . Event $e_{3}$ is not reordered and hence its cost is unchanged and is 1 . This sequence is valid because cost associated with each of the events in this sequence lies within the allowable range. Similarly, if we consider another permuted sequence $\left\{e_{3}, e_{1}, e_{2}\right\}$, then the cost associated with each of these events is $\{3,0,0\}$ which exceeds the allowable range. So, this permutation sequence is not valid. We do this for all the permuted sequences to generate the valid traces. 


\subsection{Experimental Results}

We have implemented and evaluated the quasi linearizability checking method in the PAT verification framework [20]. Our new algorithm can directly check a relaxed version of the refinement relation. This new algorithm subsumes the standard refinement checking procedure that has already been implemented in PAT. In particular, when $Q F=0$, our new procedure degenerates to the standard refinement checking procedure. When $Q F>0$, our new procedure has the added capability of checking for the quantitatively relaxed refinement relation. Our algorithm can directly handle the implementation model $M_{i m p l}$, the standard (not quasi) specification model $M_{s p e c}$, and the quasi factor $Q F$, thereby completely avoiding the user's intervention.

Table 4.2: Statistics of the benchmark examples

\begin{tabular}{|l|l|c|c|}
\hline Class & Description & Linearizable & Quasi Lin. \\
\hline Quasi Queue (3) & Segmented linked list implementation (size=3) & No & Yes \\
\hline Quasi Queue (6) & Segmented linked list implementation (size=6) & No & Yes \\
\hline Quasi Queue (9) & Segmented linked list implementation (size=9) & No & Yes \\
\hline Quasi Queue (4) & Segmented linked list implementation (size=4) & No & Yes \\
\hline Quasi Queue (8) & Segmented linked list implementation (size=8) & No & Yes \\
\hline Queue buggy1 & $\begin{array}{l}\text { Segmented queue with a bug (Dequeue on the empty } \\
\text { queue may erroneously change current segment) }\end{array}$ & No & No \\
\hline Queue buggy2 & $\begin{array}{l}\text { Segmented queue with a bug (Dequeue may get } \\
\text { value from a wrong segment) }\end{array}$ & No & No \\
\hline Lin. Queue & A linearizable (hence quasi) implementation & Yes & Yes \\
\hline Q. Priority Queue (6) & Segmented linked list implementation (size=6) & No & Yes \\
\hline Q. Priority Queue (9) & Segmented linked list implementation (size=9) & No & Yes \\
\hline Q. Priority Queue (4) & Segmented linked list implementation (size=4) & No & Yes \\
\hline Priority Queue buggy & $\begin{array}{l}\text { Segmented priority queue (Dequeue on the empty } \\
\text { priority queue may change current segment) }\end{array}$ & No & No \\
\hline Lin. Stack & A linearizable (hence quasi) implementation & Yes & Yes \\
\hline
\end{tabular}

We have evaluated our new algorithm on a set of models of standard and quasi linearizable concurrent data structures $[1,12,17]$, including queues, stacks, quasi queues, quasi stacks, and quasi priority queues. For each data structure, there can be several variants, each of which has a slightly different implementation. In addition to the implementations that are known to be linearizable 
Table 4.3: Results for checking quasi linearizability with 2 threads

\begin{tabular}{|l|c|c|c|c|}
\hline Class & QF & Verification Time (s) & Number of Visited States & Number of Visited Transitions \\
\hline Quasi Queue (3) & 2 & 7.2 & 126,810 & 248,122 \\
\hline Quasi Queue (6) & 2 & 21.2 & 237,760 & 468,461 \\
\hline Quasi Queue (9) & 2 & 114.5 & $1,741,921$ & $3,424,280$ \\
\hline Quasi Queue (4) & 3 & 131.6 & 442,558 & 869,129 \\
\hline Quasi Queue (8) & 3 & 1517.1 & $1,986,924$ & $3,754,489$ \\
\hline Queue buggy1 & 2 & 0.4 & 1,204 & 809 \\
\hline Queue buggy2 & 2 & 0.1 & 345 & 345 \\
\hline Lin. Queue & 2 & 5.5 & 240,583 & 121,548 \\
\hline Q. Priority Queue (6) & 2 & 34.3 & 472,981 & $2,905,530$ \\
\hline Q. Priority Queue (9) & 2 & 198.4 & $1,478,045$ & $2,566,427$ \\
\hline Q. Priority Queue (4) & 3 & 343.1 & $1,408,763$ & 894 \\
\hline Priority Queue buggy & 2 & 5.4 & 894 & 6,896 \\
\hline Lin. Stack & 2 & 0.2 & 2,690 & \\
\hline
\end{tabular}

and quasi linearizable, we also have versions which initially were thought to be correct, but were subsequently proved to be buggy by our verification tool. The characteristics of all benchmark examples are shown in Table 4.2. The first two columns list the name of the concurrent data structures and a short description of the implementation. The next two columns show whether the implementation is linearizable and quasi linearizable.

Table 4.3 shows the results of the experiments. The experiments are conducted on a computer with an Intel Core-i7, $2.5 \mathrm{GHz}$ processor and 8 GB RAM running Windows 7. The first column shows the statistics of the test program, including the name and the size of benchmark. The second column is the quasi factor showing the relaxation bound allowed for the model. The next three columns show the runtime performance, consisting of the verification time in seconds, the total number of visited states, and the total number of transitions made. The number of states and the running time for each of the models increase with the data size.

For 3 segmented quasi queue with quasi factor 2 , the verification completes in 7.2 seconds. It is much faster than the first approach presented in Section 4, where the same setting requires 130.7 seconds for the verification. Subsequently, as the size increases, the time to verify the quasi 
queue increases. For queue with size 6 and 9, verification is completed in 21.2 seconds and 114.5 seconds, respectively. As the quasi factor is increased to 3, the verification time for quasi queue with size 4 and 8 is increased to 131.6 seconds 1517.1 seconds respectively, which is much higher in comparison to the time for quasi factor 2 . This is basically because of the significant increment in state expansion for the higher quasi factor. For the priority queues where enqueue and dequeue operations are performed based on the priority, the verification time is higher than the regular quasi queue. Also, it is important to note that the counterexample is produced with exploration of only part of the state space for the buggy models. The verification time is much faster for the buggy queue, which shows that our approach is effective if the quasi linearizability is not satisfied. In all test cases, our method was able to correctly verify quasi linearizability or detect the violations.

\subsection{Conclusion}

In this chapter, we presented a novel approach to automate the quasi linearizability refinement checking algorithm presented in Chapter 3. The specification model is relaxed on the fly by using our new refinement checking algorithm. This makes the method more robust and less error prone as the specification is relaxed automatically as opposed to manually by the user. 


\section{Chapter 5}

\section{Conclusions and the Future Work}

In this thesis, we have presented a new method for formally verifying quasi linearizability of the implementation models of concurrent data structures. We have explored two approaches, one of which is based on manual construction of the relaxed specification model, whereas the other is fully automated, and is based on checking a relaxed version of the refinement relation between the implementation model and the specification model. The key idea of this work is to construct the quasi linearizable specification manually initially to analyze the bulkiness of the work and the cases of committing errors in modeling those specification models. This provides us a framework to extend the original refinement algorithm in order to verify the relaxed implementation model by automating the checker.

Experimental results have showed that our approach is not only able to verify the linearizable models, but also the relaxed linearizable models. The concurrent models with bugs have also 
been identified correctly in all cases. The counterexample traces generated for the buggy models clearly can provide us valuable information on why the models being buggy. Based on such information, we have been able to fix the bugs in our original models to get the correct versions of the implementation models.

The main objective of this thesis work is to develop an automated software tool that can formally verify a relaxed version of the linearizability property in the models of concurrent data structures. The main bottleneck of our approach is state space expansion, meaning the state space increases exponentially for higher number of processes as well as size of model. Also, it is limited by the fact that we can only verify the model with a bounded size to ensure that it has a finite state space. We believe that the automated refinement checking algorithm can be further optimized to improve the performance. For future work, we plan to incorporate advanced state space reduction techniques such as symmetry reduction and partial order reduction [14]. 


\section{Bibliography}

[1] Y. Afek, G. Korland, and E. Yanovsky. Quasi-Linearizability: Relaxed consistency for improved concurrency. In International Conference on Principles of Distributed Systems, pages 395-410, 2010.

[2] J. Aspnes, M. Herlihy, and N. Shavit. Counting networks. J. ACM, 41(5):1020-1048, 1994.

[3] H. Attiya and J. Welch. Distributed Computing: Fundamentals, Simulations, and Advanced Topics. John Wiley \& Sons, Inc., Publication, 2nd edition, 2004.

[4] S. Burckhardt, C. Dern, M. Musuvathi, and R. Tan. Line-up: a complete and automatic linearizability checker. In ACM SIGPLAN Conference on Programming Language Design and Implementation, pages 330-340, 2010.

[5] P. Cerný, A. Radhakrishna, D. Zufferey, S. Chaudhuri, and R. Alur. Model checking of linearizability of concurrent list implementations. In International Conference on Computer Aided Verification, pages 465-479, 2010. 
[6] A. Farzan and P. Madhusudan. Causal atomicity. In International Conference on Computer Aided Verification, pages 315-328, 2006.

[7] A. Farzan and P. Madhusudan. Monitoring atomicity in concurrent programs. In International Conference on Computer Aided Verification, pages 52-65, 2008.

[8] C. Flanagan and S. Qadeer. A type and effect system for atomicity. In ACM SIGPLAN Conference on Programming Language Design and Implementation, pages 338-349, 2003.

[9] T. A. Henzinger, A. Sezgin, C. M. Kirsch, H. Payer, and A. Sokolova. Quantitative relaxation of concurrent data structures. In ACM SIGACT-SIGPLAN Symposium on Principles of Programming Languages, 2013.

[10] M. Herlihy and J. M. Wing. Linearizability: A correctness condition for concurrent objects. ACM Trans. Program. Lang. Syst., 12(3):463-492, 1990.

[11] C. A. R. Hoare. Communicating Sequential Processes. Prentice Hall, Englewood Cliffs, NJ, 1985.

[12] C. M. Kirsch, H. Payer, H. Röck, and A. Sokolova. Performance, scalability, and semantics of concurrent fifo queues. In International Conference on Algorithms and Architectures for Parallel Processing, pages 273-287, 2012.

[13] L. Lamport. How to make a multiprocessor computer that correctly executes multiprocess programs. IEEE Trans. Computers, 28(9):690-691, 1979. 
[14] Y. Liu, W. Chen, Y. Liu, S. Zhang, J. Sun, and J. S. Dong. Verifying linearizability via optimized refinement checking. IEEE Transactions on Software Engineering, 2013.

[15] Y. Liu, W. Chen, Y. A. Liu, and J. Sun. Model checking linearizability via refinement. In Proceedings of the 2nd World Congress on Formal Methods, pages 321-337, Berlin, Heidelberg, 2009. Springer-Verlag.

[16] N. Lynch. Distributed Algorithms. Morgan Kaufmann, 1997.

[17] H. Payer, H. Röck, C. M. Kirsch, and A. Sokolova. Scalability versus semantics of concurrent fifo queues. In ACM Symposium on Principles of Distributed Computing, pages 331-332, 2011.

[18] C. Sadowski, S. N. Freund, and C. Flanagan. Singletrack: A dynamic determinism checker for multithreaded programs. In European Symposium on Programming, pages 394-409, 2009.

[19] J. Sun, Y. Liu, J. S. Dong, and C. Chen. Integrating specification and programs for system modeling and verification. In Proceedings of the third IEEE International Symposium on Theoretical Aspects of Software Engineering, pages 127-135, 2009.

[20] J. Sun, Y. Liu, J. S. Dong, and J. Pang. PAT: Towards Flexible Verification under Fairness. In Proceedings of the 21th International Conference on Computer Aided Verification, volume 5643 of Lecture Notes in Computer Science, pages 709-714, 2009. 
[21] R. K. Treiber. Systems Programming: Coping with Parallelism. Technical Report RJ 5118, IBM Almaden Research Center, 1986.

[22] V. Vafeiadis. Shape-value abstraction for verifying linearizability. In International Conference on Verification, Model Checking, and Abstract Interpretation, pages 335-348, Berlin, Heidelberg, 2009. Springer-Verlag.

[23] V. Vafeiadis, M. Herlihy, T. Hoare, and M. Shapiro. Proving correctness of highly-concurrent linearisable objects. In ACM SIGPLAN symposium on Principles and practice of parallel programming, pages 129-136, New York, NY, USA, 2006. ACM.

[24] M. T. Vechev, E. Yahav, and G. Yorsh. Experience with model checking linearizability. In International SPIN Workshop on Model Checking Software, pages 261-278, 2009.

[25] W. Vogels. Eventually consistent. Commun. ACM, 52(1):40-44, 2009.

[26] C. Wang, R. Limaye, M. Ganai, and A. Gupta. Trace-based symbolic analysis for atomicity violations. In International Conference on Tools and Algorithms for Construction and Analysis of Systems, pages 328-342, 2010.

[27] L. Wang and S. D. Stoller. Runtime analysis of atomicity for multithreaded programs. IEEE Trans. Software Eng., 32(2):93-110, 2006. 